\title{
Lieu de résidence et discrimination salariale : le cas des jeunes habitant dans une zone urbaine sensible
}

\author{
Thomas Couppié, Jean-François Giret et Stéphanie Moullet*
}

\begin{abstract}
Le but de cet article est l'étude des écarts de rémunération entre les jeunes habitant dans les zones urbaines sensibles (ZUS) à la fin de leurs études et ceux qui ne résident pas en ZUS mais qui vivent dans des unités urbaines comprenant des ZUS, en tenant compte de possibles barrières dans l'accès à certains emplois et notamment aux emplois de cadre. Dans la lignée de Brown, Moon et Zoloth (1980), nous proposons une décomposition des écarts de salaires intégrant la possibilité d'une différenciation d'accès aux emplois en fonction du type de quartier où résident les jeunes.
\end{abstract}

Les données utilisées sont issues de la première vague de l'enquête du Céreq Génération 98, portant sur les sortants du système éducatif en 1998 et interrogés en 2001. Les jeunes qui résident en ZUS à la fin de leurs études ont des rémunérations de $13 \%$ inférieures aux autres jeunes et accèdent à des emplois moins qualifiés.

La décomposition montre que plus des deux tiers de la différence salariale s'explique par des différences dans l'accès aux emplois. De plus, l'ensemble des résultats obtenus souligne le poids des faibles dotations en capital humain et en capital social des jeunes issus de ZUS, dotations qui les cantonnent dans des emplois peu qualifiés et se trouvent à l'origine de leurs plus faibles rémunérations. En revanche, les écarts de salaire que l'on pourrait expliquer par de la discrimination sont plus faibles.

Ces résultats sont néanmoins à relativiser. Nous nous focalisons sur les écarts de rémunération au premier emploi en fonction de la localisation des jeunes au moment où ils ont quitté le système éducatif. Or, ce lieu d'habitation est en partie endogène dans la mesure où il dépend des choix de résidence des parents qui peuvent être liés aux projets scolaires ou professionnels de leurs enfants. De plus, la décomposition ne permet pas de tenir compte d'un effet de sélection dans l'accès à l'emploi qui peut s'expliquer par des caractéristiques individuelles non observées.

\footnotetext{
* Thomas Couppié, appartient au Céreq, Jean-François Giret à l'Iredu (UMR CNRS 5225) - Université de Bourgogne, Stéphanie Moullet au Lest (UMR CNRS 6123) - Université de la Méditerranée. Ces deux derniers appartenaient encore au Céreq au moment de la rédaction de la première version de cet article. Nous remercions les trois rapporteurs anonymes de la revue pour leurs remarques constructives qui nous ont permis d'améliorer la version initiale de notre étude.
} 
$\mathbf{L}$ es zones urbaines sensibles (ZUS), définies comme quartiers prioritaires par le pacte de relance de la ville en 1996, cristallisent une part importante des efforts de la politique publique de la ville sous forme d'aides à la réhabilitation du parc de logements, d'aide à l'implantation ou au soutien d'activités, d'accès prioritaires à certains dispositifs d'insertion et d'accès à l'emploi, de soutien à la vie associative et à l'exercice de la citoyenneté (1). La dernière décennie a vu une forte augmentation du nombre de chômeurs dans ces zones urbaines malgré une forte baisse de la population y résidant (Le Toqueux et Moreau, 2002). La part des chômeurs dans la population active y était de $22 \%$ en 2005 , contre $10,5 \%$ dans les autres quartiers des mêmes agglomérations, soit le double de la population nationale (Pan Ké Shon, 2007) (2). Cette progression du nombre de chômeurs a été particulièrement sensible pour les jeunes résidant en ZUS, leur taux de chômage passant de $28,5 \%$ en 1990 à 38,6 \% en 2000 (contre 19,6 à 25,6\% sur l'ensemble du territoire national). De plus, une fois en emploi, il existe une surqualification des actifs des zones sensibles par rapport à leur emploi effectivement occupé (Rapport de l'Observatoire des ZUS, 2006) : moins de la moitié des jeunes diplômés du supérieur accède à des postes de cadre contre plus des deux tiers pour les actifs résidant dans les autres quartiers des unités urbaines contenant des ZUS.

Les concentrations importantes de chômeurs dans ces zones, associées à une politique du logement qui n'a pas permis de réduire les phénomènes de ségrégation spatiale, peuvent conduire à s'interroger sur le caractère stigmatisant de l'appartenance à ces quartiers. Choffel et Delattre (2003) montrent ainsi qu'à caractéristiques identiques, habiter dans une ZUS tend en moyenne à augmenter les durées de chômage. Cependant, ils observent que cet effet est plutôt modéré par rapport à celui des caractéristiques individuelles comme la formation et varie fortement d'une zone d'emploi à l'autre ou selon l'origine nationale des individus. En effet, les contraintes spatiales d'accès aux emplois se combinent souvent à d'autres difficultés des jeunes issus du ZUS. Les disparités en termes de dotation en capital humain, notamment en ce qui concerne la formation initiale, sont particulièrement fortes : malgré les politiques mises en place pour les zones d'éducation prioritaires (ZEP) (3) en particulier, les taux de réussite sont sensiblement moins élevés du fait surtout de caractéristiques initiales des jeunes très différentes (Euvrard et Rondeau, 2004). À ces difficultés des jeunes dans l'enseignement secondaire, s'ajoute un manque d'infor- mation sur l'orientation et les formations dans l'enseignement supérieur (Beaud, 2002), puis, pour certains, dans l'accès au marché du travail (Silberman et Fournier, 1994). Un certain nombre de mesures initiées ou appuyées par les pouvoirs publics, plus ou moins assimilables à des politiques de "discrimination positive », ont émergé pour favoriser l'accès des jeunes issus de ZUS à l'enseignement supérieur. C'est le cas par exemple de la charte pour l'égalité des chances dans l'accès aux formations d'excellence, impliquant trois ministères, la Conférence des Présidents d'Université, la Conférence des Grandes Écoles et la Conférence des Directeurs d'Écoles d'Ingénieurs, et permettant un accompagnement spécifique des jeunes issus de ZEP et ZUS dans ces formations. D'autres mesures dépassent le cadre de l'accès à la formation et visent également à favoriser le recrutement de jeunes issus de quartiers sensibles à des postes de responsabilité dans de grandes entreprises en proposant des stages ou en formant les diplômés à la recherche d'emploi et aux entretiens d'embauche. Dans le secteur des télécommunications, deux ministères et six grandes entreprises du secteur ont ainsi signé le « Cercle Passeport Télécoms » (4) facilitant non seulement l'accès aux formations des cadres du secteur, mais également le recrutement des jeunes issus de ZUS dans des postes à responsabilité dans le secteur et en dehors du secteur.

Nous nous proposons dans cet article d'apporter un éclairage empirique sur les conditions d'accès des jeunes issus de ZUS au marché du travail. Plus précisément, nous nous focalisons sur les écarts de rémunération entre jeunes résidant dans une ZUS à leur entrée sur le marché du travail et jeunes qui ne sont pas issus de ZUS mais qui résident dans des unités urbaines comprenant des ZUS. L'objectif est d'expliquer ces différences de salaire en tenant compte de possibles barrières dans l'accès à certains emplois et notamment aux emplois de cadres. Dans le cadre standard de la théorie du capital humain et des fonctions de gains qui en résultent, il est possible d'attribuer ces écarts soit à des différences de caractéristiques individuelles entre les jeunes vivant en ZUS et les autres, soit à des

1. Les zones urbaines sensibles, au nombre de 751 , sont définies par la loi sur la base de l'écart de leur situation sociale à la situation de l'agglomération d'appartenance.

2. Le chômage touche inégalement les jeunes hommes et les jeunes femmes : $45 \%$ des premiers sont à la recherche d'un emploi et $38 \%$ des jeunes femmes contre respectivement $22 \%$ et $23 \%$ hors ZUS de leurs agglomérations urbaines.

3. En 2001-2002, 95,5\% des élèves de collèges des ZUS sont en ZEP. Seuls $5 \%$ des ZUS ayant un collège ne scolarisent pas tous leurs élèves en ZEP.

4. Cf. http ://www.passeport-telecoms.com/. 
différences dans la valorisation salariale de ces caractéristiques, ce dernier type d'effet pouvant résulter de discriminations salariales. À la suite des travaux d'Oaxaca (1973) et de Blinder (1973), une abondante littérature économétrique s'est attachée à identifier différents facteurs dans les inégalités de salaire dont certains peuvent être expliqués par de la discrimination. Notre approche est en partie différente dans la mesure où nous prenons en compte la possibilité d'une différenciation de l'accès aux emplois en fonction du type de quartier où résident les individus. Autrement dit, les jeunes résidant dans des ZUS pourraient avoir de faibles rémunérations du fait de leur cantonnement dans des emplois peu rémunérés sur le marché du travail. Ils auraient ainsi plus de difficultés à accéder à des emplois d'encadrement qu'à des emplois non qualifiés, ce qui expliquerait leurs salaires moindres. Ces difficultés pourraient être dues à des caractéristiques défavorables, comme une plus faible dotation en capital humain ou une absence de capital social (5) et/ou à des différences de valorisation de ces caractéristiques. Dans ce dernier cas, cela pourrait correspondre à des discriminations dans l'accès aux emplois. La méthode de décomposition utilisée dans cet article a été initialement développée par Brown, Moon et Zoloth (1980) (6). Il s'agit de décomposer l'écart salarial moyen entre d'une part, ce qui est imputable aux différences de caractéristiques individuelles, et d'autre part, ce qui tient à des valorisations différentes des attributs individuels dans l'accès aux emplois puis dans la rémunération au sein de chaque emploi.

\section{Inégalités de dotations et/ou inégalités de traitement : les difficultés des jeunes issus de ZUS sur le marché du travail}

$\mathbf{L}$ 'économie urbaine se penche depuis longtemps sur les relations entre localisation résidentielle et accessibilité aux emplois. Plusieurs effets non exclusifs expliquent ces difficultés (Ihlanfeldt et Sjoquist, 1998 ; Gobillon, Selod et Zenou, 2007). Pour les habitants de certaines zones urbaines, la faible densité d'emplois dans leur voisinage - et donc l'éloignement relatif de leur lieu de résidence par rapport aux opportunités d'emplois - a un effet négatif à la fois sur l'accès aux emplois et sur la qualité des emplois trouvés. Par ailleurs, la ségrégation risque de générer des externalités sociodémographiques négatives, notamment scolaires, aggravant les difficultés de ces populations déjà défavorisées. Elle peut également accroître les difficultés d'accès à l'information sur les opportunités d'emploi. On peut enfin penser à l'existence d'une discrimination spécifiquement territoriale qui conduirait les employeurs à ne pas recruter les habitants de certaines zones.

\section{Vivre loin des emplois potentiels a des conséquences négatives sur le marché du travail}

Dans la tradition des analyses initiées par Kain (1968), émerge l'hypothèse selon laquelle l'éloignement physique entre localisation résidentielle et localisation des principaux centres d'emplois produirait un mauvais appariement spatial (« spatial mismatch »), préjudiciable aux populations les plus éloignées physiquement des emplois et qui pourrait en partie expliquer leurs difficultés sur le marché du travail. Dans ce contexte, l'éloignement induirait différents freins ou barrières à la mobilité des individus. En premier lieu, il compliquerait les possibilités de prospection du fait d'un accès réduit aux canaux d'informations sur les emplois vacants et d'une plus grande méconnaissance des employeurs potentiels (Ilhanfeldt, 1997). L'éloignement réduirait l'intensité de recherche d'emploi (Smith et Zenou, 2003). Il induirait également des coûts directs de recherche d'emploi plus élevés (Ortega, 2000). Enfin, il réduirait l'attractivité des emplois prospectés et des salaires attendus du fait de coûts attendus (monétaires et/ou temporels) de migrations alternantes (Coulson, Laing et Wang, 2001).

Les conséquences sur le marché du travail sont alors multiples. Le mauvais appariement spatial implique des opportunités d'emploi réduites pour les groupes d'individus résidant dans les zones éloignées des centres d'emplois. Cette réduction des opportunités engendre à son tour, au sein de ces zones résidentielles, une concurrence accrue pour les emplois accessibles, concurrence susceptible de limiter l'accès à l'emploi d'une partie des individus mais aussi d'induire des processus spécifiques de déclassement (Büchell et Van Ham, 2003) et une pression accrue sur les salaires (Raphael, 1998 ; Brueckner et Zenou, 2003). De plus, compte tenu des mécanismes de localisation des différents types d'entreprises et de l'existence

5. C'est-à-dire, une absence ou une faiblesse de réseaux sociaux.

6. Pour une application sur données françaises, voir notamment (Meng et Meurs, 2001) sur les écarts salariaux entre hommes et femmes. 
de procédures de recrutement différenciées pour les différentes catégories d'emploi, ce mauvais appariement spatial apparaît davantage préjudiciable aux individus peu ou pas diplômés dont les cibles professionnelles correspondent principalement aux catégories d'emplois peu qualifiés, dans l'industrie et dans les services (Turner, 1997 ; Raphael, 1998 ; Hellerstein, Neumark et McInerney, 2008). En France, Gobillon et Selod (2004 et 2007) montrent par exemple que dans la région parisienne, les indices de dissimilarités entre lieux de résidence et d'emploi varient largement selon la catégorie socioprofessionnelle des emplois, le niveau de diplôme des individus ou leur origine nationale et vont affecter les chances de sortie du chômage.

\section{La ségrégation socio-spatiale vue comme un processus inégalitaire d'accumulation de capital humain}

Comme le souligne Borjas (1995) dans le cas des populations immigrées, les phénomènes de ségrégation urbaine sont des éléments déterminants dans l'acquisition de capital humain et dans le processus de mobilité intergénérationnelle. Si la formation scolaire est un des principaux vecteurs de cette différenciation, les modes d'accumulation du capital humain vont aussi dépendre de facteurs environnementaux beaucoup plus larges. Ainsi, Becker (1993) souligne dans sa présentation du capital humain que l'environnement familial pèse grandement sur les savoirs, les habitudes, les valeurs que les enfants peuvent acquérir : les relations sociales à l'intérieur de la famille, la langue parlée à la maison ou l'aide accordée à la maîtrise de la langue peuvent conférer aux jeunes un avantage dans l'apprentissage des savoirs scolaires et extra-scolaires. Cependant, le rôle joué par l'environnement dépasse le seul cadre familial (Borjas, 1995) : les effets de voisinage, de quartiers peuvent également influencer l'acquisition de capital humain. En France, Goux et Maurin (2005) montrent que ces effets de voisinage vont influencer et même amplifier, pour les nouveaux arrivants, les différences de résultats scolaires des enfants, même dans le cas où les niveaux de ressources des familles sont proches.

\section{Un système informationnel sur le marché du travail moins adapté pour les jeunes résidant dans les quartiers socialement défavorisés}

L'acquisition d'informations sur les opportunités d'emplois constitue également un élément central pour expliquer les différences d'accès à l'emploi notamment dans le cas de fortes ségrégations urbaines (Ioannides et Loury, 2004). La concentration de la main-d'œuvre immigrée sur certaines activités économiques conduit à une forte polarisation de leurs réseaux sociaux (Borjas, 1992), transmissibles en partie à leurs enfants. Dans ce cas, les explications sont souvent liées à des phénomènes de concentration ethnique ou de familles immigrées de la première ou de la seconde génération provenant de pays différents. Loury (2006) distingue ainsi les discriminations concernant l'accès à l'emploi et les discriminations en termes de contacts, qui correspondent en fait aux différences d'accès aux informations et réseaux sociaux. Comme le montrent en France Silberman et Fournier (1999) à partir d'enquêtes du Céreq sur les sortants du système scolaire, des différences existent dans la mobilisation des réseaux sociaux pour les enfants des familles immigrées : lors de leurs premiers emplois, ceux-ci sont globalement un peu moins nombreux que les autres à travailler dans des entreprises où ils connaissent quelqu'un. L'éloignement géographique vient alors renforcer les coûts de recherche d'emploi lorsque les réseaux sociaux permettant d'accéder aux emplois sont peu ou pas présents. L'existence de dispositifs institutionnels permettant d'aider les jeunes dans la recherche d'emploi peut compenser en partie les difficultés d'accès aux informations directes sur les opportunités d'emploi. Cependant, là encore, des travaux plus qualitatifs pointent plutôt une « défaillance des dispositifs d'insertion sociale et professionnelle pour les jeunes issus des ZUS » (De Stéphanis, Mathey-Pierre et Dugué, 2004).

\section{Des pratiques discriminatoires sur le marché du travail liées à la stigmatisation du quartier}

Les différences de dotation en capital humain ou l'absence de réseau, d'information sur l'emploi ne sont pour autant pas la seule explication des difficultés des jeunes issus de quartiers sensibles sur le marché du travail. Se pose notamment la question de la valorisation de leurs caractéristiques scolaires. Les phénomènes de discrimination territoriale, conséquence de pratique de recrutement des employeurs, sont parfois désignés comme une cause des difficultés des jeunes issus de certains quartiers dans l'accès aux emplois. Dans la vision initiale de la discrimination développée dans les travaux de Becker (1957), cette dernière était la conséquence de « préférences discriminatoires » de 
la part des employeurs à l'encontre de certains groupes en général minoritaires. La théorie de la discrimination statistique développée par Phelps (1972) est au contraire fondée sur un jugement statistique des employeurs : l'imperfection de l'information sur le marché du travail et sur la productivité des candidats à l'embauche conduit les employeurs à « trier » des individus en fonction de certaines caractéristiques. Dans ce cas, les caractéristiques retenues pour sélectionner les individus devront être corrélées avec la productivité réelle des candidats à l'embauche. L'employeur pourra par exemple sélectionner les individus en fonction de ses observations sur la productivité moyenne du groupe associé à chaque caractéristique : la discrimination découle alors du manque d'information sur les caractéristiques des candidats à l'embauche. Il peut alors exister une discrimination territoriale si les employeurs attribuent aux individus dont la productivité n'est pas observée la productivité moyenne des travailleurs habitant dans le quartier (Zenou, 2002).

\section{Une méthode de décomposition de l'écart de salaire prenant en compte les barrières d'accès aux emplois qualifiés}

Uite aux travaux d'Oaxaca et de Blinder, une $\checkmark$ abondante littérature économétrique s'est attachée à identifier les différentes composantes des écarts de salaires, dont celle relative à la discrimination. Les méthodes utilisées occultent souvent l'hypothèse que cette discrimination salariale puisse être indirecte et passer par une inégalité dans les possibilités d'accès aux différentes catégories d'emploi. Or, comme on vient de le voir, il peut exister pour les habitants de certaines aires géographiques des barrières dans l'accès à certains types d'emploi, qui peuvent in fine expliquer les différences de rémunération.

Les travaux de l'Observatoire National des Zones Urbaines Sensibles montrent que les jeunes issus de ZUS ont des rémunérations inférieures aux autres et se trouvent cantonnés dans des emplois peu rémunérés (Observatoire National des Zones Urbaines Sensibles, 2006). Ils ont notamment plus de difficultés à accéder à des emplois d'encadrement qu'à des emplois non qualifiés, expliquant en partie de plus faibles rémunérations perçues. Ces difficultés peuvent être dues à des caractéristiques individuelles défavorables, comme par exemple leur moindre dotation en capital humain ou en capital social mais également à des différences de valorisation de ces caractéristiques. Dans ce dernier cas, on peut parler de discrimination dans l'accès aux catégories d'emplois.

La méthodologie proposée par Brown, Moon et Zoloth (1980) présente l'intérêt d'ajouter à la décomposition de type Oaxaca-Blinder, des facteurs explicatifs liés à l'accès aux différentes catégories d'emplois. Rappelons que le principe de la méthode d'Oaxaca-Blinder est de distinguer dans l'écart de salaire moyen entre deux populations une composante associée aux différences de caractéristiques explicatives des rémunérations (part dite « expliquée ») et une composante liée aux différences de rendements de ces caractéristiques (part dite «non expliquée », sous-entendue par les différences de caractéristiques). Retenir la méthode de Brown, Moon et Zoloth permet d'isoler de nouvelles composantes du différentiel salarial. En prenant en compte l'accès aux différentes catégories d'emploi, il s'agit de distinguer les parts «expliquée » et " inexpliquée » de l'écart de gain liées au fait que les individus des deux populations n'ont pas les mêmes emplois (écart « inter-catégorie ») des parts « expliquée » et « inexpliquée » liées aux différences faites à l'intérieur de chaque catégorie d'emploi (écart « intra-catégorie »).

L'idée de base est d'utiliser la structure de l'emploi des jeunes résidant hors d'une ZUS et les rémunérations associées à ces emplois comme une norme pour prédire la structure hypothétique des emplois des jeunes originaires de ZUS et leurs rémunérations moyennes s'il n'y avait pas de « discrimination » (c'est-à-dire de préférence pour les jeunes n'habitant pas dans une ZUS dans l'accès à l'emploi). Cela revient à prédire les emplois et à estimer les salaires qu'auraient les jeunes de ZUS si leurs caractéristiques individuelles déterminaient leurs emplois (et leurs salaires associés) à l'instar de ce que l'on observe pour les jeunes « hors ZUS » (HZUS).

Cet écart de salaire entre " hors ZUS » et ZUS peut s'écrire de la manière suivante (cf. annexe 1) :

$$
\begin{aligned}
& \bar{W}_{H Z U S}-\bar{W}_{Z U S}=\sum_{j} P_{j}^{Z U S} \hat{\beta}_{j}^{*}\left(\bar{X}_{j}^{H Z U S}-\bar{X}_{j}^{Z U S}\right) \\
& +\sum_{j} P_{j}^{Z U S} \bar{X}_{j}^{H Z U S}\left(\hat{\beta}_{j}^{H Z U S}-\hat{\beta}_{j}^{*}\right)+ \\
& \sum_{j} P_{j}^{Z U S} \bar{X}_{j}^{Z U S}\left(\hat{\beta}_{j}^{*}-\hat{\beta}_{j}^{Z U S}\right)
\end{aligned}
$$




$$
\begin{aligned}
& +\sum_{j} \bar{W}_{j}^{H Z U S}\left(P_{j}^{H Z U S}-\hat{P}_{j}^{Z U S}\right) \\
& +\sum_{j} \bar{W}_{j}^{H Z U S}\left(\hat{P}_{j}^{Z U S}-P_{j}^{Z U S}\right)
\end{aligned}
$$

$\bar{W}$ est le salaire moyen estimé, $\hat{\beta}_{j}^{H Z U S}$ et $\hat{\beta}_{j}^{Z U S}$ sont les coefficients estimés des équations de salaires (les rendements des caractéristiques individuelles) pour les jeunes issus de ZUS et ceux « hors ZUS » pour les $j$ catégories d'emploi retenues. La méthode suppose donc que l'on estime une équation de salaire pour chaque population $l$ (ZUS et HZUS) et pour chaque catégorie d'emploi $j=1, \ldots J$.

La différence de salaire moyen entre les jeunes de ZUS et ceux résidant hors de ces zones (au sens de notre définition) se décompose donc en cinq termes (cf. tableau 1).

Les termes (1), (2) et (3) forment la part de l'écart de salaire entre les ZUS et les HZUS qui relève des différences de rémunération à l'intérieur des catégories d'emploi (écart intracatégorie). Les termes (4) et (5) forment l'écart inter-catégorie.

Au total, une partie de l'écart de salaire entre les jeunes de ZUS et " hors ZUS » est expliquée par des écarts dans les caractéristiques que l'on retient (écart expliqué : (1) + (4)), l'autre partie est non expliquée car elle ne relève pas de ces différences d'attributs (écart non expliqué : (2) $+(3)+(5))$. Dans les travaux de décomposition, ces termes relatifs à l'écart « non expliqué » sont souvent associés à la discrimination. Soulignons que cette interprétation usuelle en termes de dis- crimination doit être comprise dans un sens plus large. En effet, la partie des caractéristiques individuelles inobservables qui influence les niveaux de salaires et qui n'est pas prise en compte dans les équations de gains pèse sur l'évaluation des rendements salariaux des caractéristiques observées. En conséquence, les écarts de rendements des caractéristiques observables vont comprendre, outre les effets de la discrimination " pure ", les effets des caractéristiques inobservables.

Ainsi, cette méthodologie permet de rendre compte de possibles phénomènes de barrières spécifiques dans l'accès à certaines catégories d'emplois, de cadres notamment. En revanche, une limite de la décomposition est de ne pas tenir compte d'un effet de sélection dans l'accès à l'emploi qui peut s'expliquer par l'hétérogénéité individuelle et notamment par des caractéristiques non observées.

Par la suite, seront introduits dans les différentes fonctions de gains et dans le modèle d'allocation des emplois (pour les estimations, cf. annexe 2) :

- des caractéristiques du parcours de formation du jeune : le plus haut diplôme obtenu à la sortie du système éducatif, la spécialité de sa formation sous une forme agrégée (spécialité industrielle versus spécialité tertiaire ou générale), le fait d'avoir effectué au moins un stage durant la formation initiale ;

- une variable indicatrice pour le sexe du jeune ;

- la profession du père qui peut être considérée comme un indicateur du capital social, ainsi que le lieu de naissance des parents ;

\section{Tableau 1}

\begin{tabular}{|c|c|}
\hline \multicolumn{2}{|c|}{ Écart de gain lié aux différences internes à chaque catégorie d'emploi } \\
\hline$\sum_{j} P_{j}^{Z U S} \hat{\beta}_{j}^{*}\left(\bar{X}_{j}^{\text {HZUS }}-\bar{X}_{j}^{Z U S}\right)$ & ... des différences de caractéristiques individuelles dans les emplois que l'on considère ; \\
\hline$\sum_{j} P_{j}^{Z U S} \bar{X}_{j}^{H Z U S}\left(\hat{\beta}_{j}^{H Z U S}-\hat{\beta}_{j}^{*}\right)$ & $\begin{array}{l}\text {... des différences de rétributions salariales (ou de "valorisation ") des caractéristiques des deux } \\
\text { populations de jeunes, à l'avantage de celle qui réside en dehors des ZUS; }\end{array}$ \\
\hline$\sum_{j} P_{j}^{Z U S} \bar{X}_{j}^{Z U S}\left(\hat{\beta}_{j}^{*}-\hat{\beta}_{j}^{Z U S}\right)$ & $\begin{array}{l}\text {... des différences de rétributions salariales (ou de "valorisation ") des caractéristiques des deux } \\
\text { populations de jeunes, au désavantage des jeunes issus de ZUS; }\end{array}$ \\
\hline \multicolumn{2}{|c|}{ Écart de gain lié au fait que les individus des deux populations n'ont pas les mêmes emplois } \\
\hline$\sum_{j} \bar{W}_{j}^{H Z U S}\left(P_{j}^{H Z U S}-\hat{P}_{j}^{Z U S}\right)$ & $\begin{array}{l}\text {... de la différence dans la structure des emplois entre les deux populations résultant unique- } \\
\text { ment du fait que les deux groupes de jeunes ont des caractéristiques individuelles différentes ; }\end{array}$ \\
\hline$\sum_{j} \bar{W}_{j}^{\text {HZUS }}\left(\hat{P}_{j}^{Z U S}-P_{j}^{Z U S}\right)$ & $\begin{array}{l}\text {... du traitement différent des jeunes de ZUS de ceux non issus d'une ZUS dans l'accès aux six } \\
\text { catégories d'emploi retenues. }\end{array}$ \\
\hline
\end{tabular}

\section{Les contributions à l'explication de l'écart des salaires moyens}


- la localisation du jeune à la fin de ses études en distinguant l'Île-de-France de la province ;

- le ratio du nombre d'emplois de cadre et de profession intermédiaire et du nombre d'emplois d'employé et d'ouvrier dans le quartier de résidence, proxy de l'environnement des jeunes, du milieu économique et social de leur voisinage.

\section{Des données portant sur les sortants du système éducatif en 1998}

$\mathbf{L}$ es données utilisées sont issues de la première vague de l'enquête du Céreq
Génération 98, portant sur les sortants du système éducatif en 1998 et interrogés en 2001 (cf. encadré). Grâce à un partenariat avec la Délégation Interministérielle à la Ville (DIV), le géocodage de l'adresse du domicile des jeunes enquêtés au moment où ils terminent leurs études a pu être réalisé (Couppié et Gasquet, 2006). Trois catégories de localisation ont été identifiées : un domicile situé dans une zone urbaine sensible (ZUS), un domicile situé hors d'une ZUS mais dans une agglomération contenant une ZUS et un domicile situé dans une zone rurale ou une agglomération urbaine dépourvue de ZUS. Afin de comparer des populations accédant au marché du travail ayant des caractéristiques relativement proches, les jeunes qui résident dans des zones rurales ou dans des unités urbaines ne contenant aucune ZUS sont

Encadré

\section{L’ENQUÊTE GÉNÉRATION 98}

Le Centre d'études et de recherches sur les qualifications (Céreq) organise régulièrement des enquêtes chargées d'observer les conditions d'insertion professionnelle des jeunes quittant le système éducatif. Dans ce cadre, il a interrogé au printemps 200155000 des 742000 jeunes sortis en 1998 des différentes filières de formation initiale pour entrer sur le marché du travail. Cette enquête, baptisée Génération 98, a permis de rassembler :

- des données individuelles longitudinales sur les parcours professionnels décrivant les épisodes d'emploi, de chômage, de formation, etc. rencontrés (trois années reconstituées) ;

- des informations concernant les caractéristiques socio-démographiques des jeunes (niveau de diplôme, catégorie sociale et pays de naissance de leurs parents (et leur nationalité), modes d'habitat, etc.) ;

- des informations à caractère subjectif sur le parcours professionnel (éléments de satisfaction professionnelle, perspectives d'avenir, sentiment de discrimination, etc.).

- Au printemps 2006, le Céreq et la Délégation interministérielle à la ville (DIV) ont noué un partenariat qui a permis de géocoder l'adresse du domicile des jeunes au moment où ils achevaient leurs études. Trois catégories de localisation ont été identifiées : un domicile situé dans une zone urbaine sensible (ZUS), un domicile situé hors d'une ZUS mais dans une agglomération urbaine contenant une ZUS et un domicile situé dans une zone rurale ou une agglomération urbaine dépourvue de ZUS. Cette étude s'appuie sur l'exploitation spécifique de l'enquête Génération 98 enrichie de ces informations.

\section{Les résultats de l'opération de géocodage}

- On estime à $6,9 \%$ la part des jeunes de la Génération 98 qui résidaient dans une zone urbaine sensible au moment de la fin de leur formation initiale en 1998. Ce taux est inférieur à ce que l'on pouvait attendre ; bien qu'on ne dispose pas d'éléments strictement comparables, rappelons que les ZUS accueillaient alors environ $8 \%$ de la population métropolitaine et que cette population est relativement jeune (Observatoire National des Zones Urbaines Sensibles, 2004). L'enquête Emploi de l'Insee estime, pour sa part, que 8,6 \% des jeunes de la cohorte de sortants 1998 résident en 2004 en ZUS (calcul des auteurs). Une opération de géocodage d'un échantillon d'adresses de non-répondants montre que cette sous-estimation des jeunes qui résidaient en ZUS à la fin de leurs études s'explique :

- pour partie par le fait que les jeunes résidant à la fin de leurs études dans des grandes unités urbaines contenant des ZUS répondent moins bien à l'enquête (51\% des répondants, $59 \%$ des non-répondants) que les jeunes résidant dans des unités urbaines dépourvues de ZUS ou en milieu rural ;

- pour partie par le fait que, au sein des grandes unités urbaines contenant des ZUS, les jeunes qui résidaient en ZUS participent un peu moins à l'enquête que les autres puisqu'ils représentent $11,5 \%$ des répondants de ces unités urbaines et $13,1 \%$ des nonrépondants. Cependant, une comparaison des caractéristiques socio-démographiques recueillies dans les deux enquêtes montre que les principales spécificités des jeunes qui résidaient en ZUS sont conservées d'une enquête à l'autre. À titre d'exemple, dans l'enquête Génération 98, un jeune qui résidait en ZUS à la fin de ses études a respectivement 2,7 et 1,2 fois plus de chances (mesurées par des rapports de cotes ou odds-ratio) de sortir sans diplôme ou avec un CAPBEP qu'un jeune résidant hors d'une ZUS (2,3 et 1,3 fois plus de chances dans l'enquête Emploi 2004). À l'inverse, un jeune qui résidait en ZUS a deux fois moins de chances de sortir avec un diplôme de l'enseignement supérieur dans l'enquête Génération 98 comme dans l'enquête Emploi 2004. 
écartés de l'analyse. Le champ retenu correspond donc à l'ensemble des jeunes sortants du système éducatif en 1998 et résidant à cette date dans une unité urbaine contenant des ZUS - et ayant eu au moins un emploi au cours de leurs trois premières passées sur le marché du travail. $\mathrm{Au}$ sein de la population retenue, près de $12 \%$ des jeunes résidaient en ZUS à la fin de leurs études.

Jeunes ayant terminé leurs études en ZUS et autres jeunes urbains au sens retenu dans cet article, c'est-à-dire résidant dans des unités urbaines contenant des ZUS, présentent des différences assez sensibles en termes de capital humain mais également de capital socioculturel - origines sociale et nationale - (cf. tableau 2). Ainsi, 26,7 \% des jeunes qui résidaient en ZUS sont non diplômés, contre $11,5 \%$ de leurs voisins jeunes urbains vivant hors des ZUS. Inversement, seulement $12,4 \%$ d'entre eux ont quitté le système éducatif avec un diplôme supérieur ou égal à $\mathrm{Bac}+4$ contre $28,5 \%$ des autres. De même, en termes de capital social, seulement 9,5\% des jeunes de ZUS ont, à la fin de leurs études, un père cadre contre $25,2 \%$ pour les jeunes urbains ne résidant pas en ZUS. Enfin, 55,1\% des jeunes résidant en ZUS ont leur père qui est né en France ou de nationalité française contre $83,1 \%$ pour les jeunes issus d'unité urbaine hors ZUS.

Concernant la région de résidence à la fin des études, la répartition entre Île-de-France et province est la même pour les jeunes résidant en ZUS que pour les autres (respectivement $22-78 \%$ pour les jeunes issus de ZUS et $23-77 \%$ pour les autres jeunes urbains). Pour autant, l'environnement socio-économique de ces quartiers diffère assez fortement. Le ratio entre le nombre de personnes employées comme cadres et professions intermédiaires et le nombre de personnes occupant des postes d'ouvrier et d'employés (7), pointe de fortes différences entre les deux types de quartier. Dans les quartiers rattachés à une ZUS, les personnes occupant des emplois de cadres et professions intermédiaires représentent près d'un tiers des emplois d'ouvriers et d'employés. Dans les quartiers urbains n'appartenant pas à des ZUS, ce rapport est presque équilibré avec neuf cadres ou professions intermédiaires pour dix ouvriers ou employés. Notons que l'indicateur est basé sur le lieu de résidence des individus : il rensei-

7. Cet indicateur est calculé au niveau du quartier de résidence (codification IRIS) à partir des données du recensement de la population de 1999

Tableau 2

Caractéristiques de la population des jeunes domiciliés en et hors d'une ZUS à la fin de leurs études en 1998

\begin{tabular}{|c|c|c|}
\hline & Jeunes issus de ZUS & Jeunes non issus de ZUS \\
\hline Homme & 52,1 & 51,2 \\
\hline $\begin{array}{l}\text { Niveau de formation initiale } \\
\text { Sans diplôme } \\
\text { CAP BEP } \\
\text { Bac } \\
\mathrm{Bac}+2 \\
\mathrm{Bac}+3 \\
\mathrm{Bac}+4 \text { et plus }\end{array}$ & $\begin{array}{r}26,7 \\
23,8 \\
18,6 \\
13,6 \\
4,9 \\
12,4\end{array}$ & $\begin{array}{r}11,5 \\
16,1 \\
17,0 \\
19,3 \\
7,6 \\
28,5\end{array}$ \\
\hline $\begin{array}{l}\text { Spécialité industrielle de formation } \\
\text { Mère née en France ou de nationalité française } \\
\text { Père né en France ou de nationalité française }\end{array}$ & $\begin{array}{l}33,2 \\
58,1 \\
55,1\end{array}$ & $\begin{array}{l}27,7 \\
84,7 \\
83,1\end{array}$ \\
\hline $\begin{array}{l}\text { CS du père à la fin des études } \\
\text { Artisan, commerçant } \\
\text { Cadre } \\
\text { Technicien et employé } \\
\text { Ouvrier } \\
\text { Inactif, chômeur }\end{array}$ & $\begin{array}{r}8,0 \\
9,5 \\
31,1 \\
35,8 \\
15,1\end{array}$ & $\begin{array}{l}11,1 \\
25,2 \\
34,7 \\
17,9 \\
11,0\end{array}$ \\
\hline $\begin{array}{l}\text { Travail à temps plein } \\
\text { Premier emploi dans le secteur public } \\
\text { Résidence en Île-de-France en fin d'études } \\
\text { Nombre de cadres et professions intermédiaires / } \\
\text { Nombre d'employés et d'ouvriers dans le quartier }\end{array}$ & $\begin{array}{r}74,4 \\
17,4 \\
21,6 \\
0,31\end{array}$ & $\begin{array}{r}77,9 \\
21,0 \\
23,3 \\
0,91\end{array}$ \\
\hline $\begin{array}{l}\text { Distance moyenne entre la commune de résidence en fin d'études et } \\
\text { celle du premier emploi (en } \mathrm{km})\end{array}$ & 38,5 & 64,0 \\
\hline
\end{tabular}

Lecture : 26,7 \% des jeunes issus d'une ZUS n'ont aucun diplôme, contre 11,5 \% pour les jeunes résidant hors d'une ZUS.

Champ : jeunes sortis du système éducatif en 1998 résidant en ZUS (première colonne) ou dans une unité urbaine contenant une ZUS (deuxième colonne) ayant eu au moins un emploi au cours de leurs trois premières années de vie active.

Source : enquête Génération 98, première interrogation, Céreq. 
gne donc moins sur la demande de travail locale que sur l'offre de travail locale, les emplois occupés pouvant se situer en dehors du quartier. Il capte par conséquent des effets de voisinage liés entre autres au capital social existant dans les quartiers. Cet indicateur peut être également considéré comme un proxy du type d'emploi auquel les jeunes peuvent accéder sans avoir à quitter leur quartier. Le calcul de la distance moyenne entre la commune de résidence des jeunes à la fin de leurs études et la commune de leur premier emploi montre que les jeunes issus de ZUS trouvent un premier emploi dans une aire géographique plus resserrée que les autres urbains.

Notre préoccupation principale porte sur les écarts de rémunération entre les deux groupes de jeunes à l'embauche du premier emploi obtenu après la fin des études. En effet, nous faisons l'hypothèse ici que les jeunes ne peuvent « choisir » leur localisation de résidence tant qu'ils n'ont pas accédé à un premier emploi et acquis leur autonomie financière, « choix » qui introduirait une dimension encore plus endogène de la localisation du lieu de résidence (8). Il n'est cependant pas exclu qu'il ait existé des choix préalables de localisation de la part des parents en fonction de leurs caractéristiques inobservables qui sont elles-mêmes corrélées avec celles de leurs enfants (9).

Les jeunes issus de ZUS perçoivent à leur première embauche un salaire mensuel de $13 \%$ inférieur à celui des jeunes habitant hors d'une ZUS. Cet écart dépend de la catégorie sociale de l'emploi que les jeunes occupent (cf. tableau 3). En particulier, l'écart de rémunération est le plus important au sein des catégories de cadres, de professions intermédiaires et d'employés qualifiés. Parmi les ouvriers qualifiés et non qualifiés et les employés non qualifiés, les différences de salaire sont quasi-inexistantes du fait de la proximité des rémunérations moyennes avec le salaire minimum.

À ces différences de rémunération, s'ajoute un inégal accès aux différentes catégories d'emploi. Selon leur lieu de résidence en fin d'études (ZUS, hors ZUS), les jeunes sont inégalement répartis dans les six catégories d'emplois (cf. tableau 4). Les emplois de cadres concernent trois fois plus souvent les résidents hors ZUS $(20,2 \%)$ que les autres $(7,8 \%)$. Les professions intermédiaires concentrent $27,4 \%$ des effectifs des résidents hors ZUS alors qu'ils n'occupent que $21,4 \%$ des jeunes issus de ZUS. Inversement, les emplois d'ouvriers non qualifiés sont occupés par $25,2 \%$ des jeunes résidents en ZUS alors qu'ils concernent seulement

8. Cette dimension endogène a d'ailleurs été suggérée dans différents travaux en France (Arrighi, Gasquet et Roux, 2008, Couppié et Gasquet, 2009, Pan Ké Shon, 2009).

9. Des parents qui habitaient en ZUS ont pu par exemple déménager pour faciliter le parcours scolaire et les projets professionnels de leurs enfants. Nous renvoyons à Aslund, Östh et Zenou (2006) ou à Raphael (1998) pour une discussion plus approfondie sur ces questions d'endogénéité.

\section{Tableau 3}

Salaires d'embauche mensuels moyens au premier emploi selon le lieu de résidence à la fin des études et selon la catégorie d'emploi

\begin{tabular}{|c|c|c|c|c|c|}
\hline & \multicolumn{2}{|c|}{$\begin{array}{c}\text { Salaire mensuel net moyen } \\
\text { (en euros de l'année 1998) } \\
\text { des jeunes qui, à leur sortie } \\
\text { du système éducatif... }\end{array}$} & \multirow{2}{*}{$\begin{array}{c}\text { Rapport de } \\
\text { salaire (1) / (2) } \\
\text { (en \%) }\end{array}$} & \multirow{2}{*}{$\begin{array}{l}\text { Différence de } \\
\text { salaire } \\
(\text { en \%) }\end{array}$} & \multirow{2}{*}{$\begin{array}{l}\text { Part des jeunes } \\
\text { résidant en ZUS } \\
\text { (en \%) }\end{array}$} \\
\hline & $\begin{array}{l}\text { en résident } \\
\text { en ZUS } \\
\text { (1) }\end{array}$ & $\begin{array}{c}\text {... résident } \\
\text { hors d'une ZUS } \\
\text { (2) }\end{array}$ & & & \\
\hline Ouvriers non qualifiés & 851 & 875 & 97,3 & 2,8 & 27,6 \\
\hline Ouvriers qualifiés & 895 & 911 & 98,2 & 1,8 & 13,7 \\
\hline Employés non qualifiés & 696 & 701 & 99,3 & 0,7 & 20,5 \\
\hline Employés qualifiés & 826 & 893 & 92,5 & 8,1 & 12,5 \\
\hline Professions intermédiaires & 982 & 1040 & 94,4 & 5,9 & 20,5 \\
\hline Cadres & 1443 & 1517 & 95,1 & 5,1 & 5,3 \\
\hline Ensemble & 881 & 996 & 88,5 & 13,0 & 11,1 \\
\hline Effectifs dans l'échantillon & 2525 & 20492 & & & \\
\hline
\end{tabular}

Lecture : lors de leur première embauche, le salaire mensuel moyen des jeunes résidant en ZUS à la sortie du système éducatif et ayant accédé à un emploi d'ouvrier non qualifié est de $851 €$ alors que, pour ce type d'emploi, celui des jeunes urbains ne vivant pas dans des ZUS est de $875 €$, soit une différence de 2,8\%.27,6 \% des jeunes ouvriers non qualifiés ont résidé dans une ZUS.

Champ : jeunes sortis du système éducatif en 1998 résidant en ZUS (première colonne) ou dans une unité urbaine contenant une ZUS (deuxième colonne) ayant eu au moins un emploi au cours de leurs trois premières années de vie active.

Source : enquête Génération 98, première interrogation, Céreq. 
$14,2 \%$ des jeunes habitant dans les autres quartiers urbains.

Il est possible dans un premier temps de tester « toutes choses égales par ailleurs » l'existence d'un effet propre lié au fait de résider dans un quartier sensible, sur la probabilité d'accès à une catégorie d'emploi donnée et sur la rémunération (cf. annexe 2, tableaux $B$ et $C$, et également Couppié et Gasquet, 2006). Une fois introduites les caractéristiques individuelles et le lieu de résidence, on montre que le fait d'habiter en ZUS s'avère être un handicap pour accéder aux catégories de cadres et de professions intermédiaires et réduit le niveau de salaire attendu. Cependant, l'effet estimé, notamment sur les salaires, est nettement inférieur à l'écart salarial observé, ce qui plaide pour un rôle important des caractéristiques individuelles dans la constitution des écarts salariaux et des distributions inégales au sein des catégories d'emploi.

Ces premiers constats illustrent les difficultés qu'ont les jeunes issus de ZUS pour accéder aux emplois les plus qualifiés et les mieux rémunérés. Ils peuvent être mis en regard avec une appréciation plus subjective des difficultés relatives à leur insertion professionnelle à travers l'expression du sentiment d'avoir été discriminé à l'embauche au cours de leur recherche d'emploi. Une question sur cette appréciation posée également dans l'enquête Génération 98, sur le même champ que celui utilisé dans cet article montre que globalement, $23 \%$ des jeunes issus de ZUS contre $13 \%$ des jeunes issus d'autres quartiers urbains affirment avoir subi une discrimination de cet ordre. De plus, le motif de cette discrimination est explicitement relié dans un certain nombre de cas à la localisation de leur domicile ; ainsi, $5 \%$ des jeunes issus de ZUS déclarent que la discrimination était du fait de leur quartier de résidence, contre $1 \%$ pour les autres jeunes.

Au-delà de la perception des individus enquêtés, en partie subjective, la méthode d'analyse développée ici - une décomposition des écarts salariaux proposée par Brown et al. (1980) - présente l'intérêt d'attribuer les écarts de rémunérations entre les jeunes issus de ZUS et les autres jeunes urbains à plusieurs types d'explication. La différence salariale peut relever de différences entre certaines caractéristiques des deux populations de jeunes (le type de diplôme ou l'origine sociale). L'écart de salaire peut également tenir à ce que les jeunes issus de ZUS accèdent moins souvent que les autres à des emplois plus qualifiés. En fin de compte, l'écart salarial se décomposera entre ce qui tient à la différence de rémunération au sein d'une même catégorie d'emploi et ce qui tient aux différences individuelles dans l'accès aux emplois.

Tableau 4

Structure des premiers emplois occupés pour les jeunes de ZUS et hors ZUS

\begin{tabular}{|l|c|c|c|}
\hline & $\begin{array}{c}\text { Structure observée } \\
\text { pour les jeunes } \\
\text { issus de ZUS }\end{array}$ & $\begin{array}{c}\text { Structure observée } \\
\text { pour les jeunes } \\
\text { non issus de ZUS }\end{array}$ & $\begin{array}{c}\text { Structure prédite } \\
\text { pour les jeunes issus de ZUS à partir } \\
\text { des coefficients estimés pour les } \\
\text { jeunes non issus de ZUS }\end{array}$ \\
\hline Ouvriers non qualifiés & 25,2 & 14,2 & 24,4 \\
& $(0,9)$ & $(0,2)$ & 13,4 \\
\hline Ouvriers qualifiés & 13,5 & 9,5 & $(0,5)$ \\
\hline Employés non qualifiés & $(0,7)$ & $(0,2)$ & 21,5 \\
\hline Employés qualifiés & 19,8 & 15,5 & $(0,6)$ \\
\hline Professions intermédiaires & $(0,8)$ & $(0,3)$ & 12,4 \\
& 12,2 & 13,2 & $(0,4)$ \\
\hline Cadres & $(0,6)$ & $(0,2)$ & 20,2 \\
& 21,4 & 27,4 & $(0,5)$ \\
\hline & $(0,8)$ & $(0,3)$ & 8,1 \\
$(0,4)$ \\
\hline
\end{tabular}

Lecture : lors de leur première embauche, 25,2 \% des jeunes résidant en ZUS à la fin de leurs études sont des ouvriers non qualifiés, ils seraient $24,4 \%$ dans cette catégorie d'emploi s'ils y étaient affectés selon les mêmes modalités que les jeunes habitant hors d'une ZUS. Seulement 14,2\% des jeunes résidant hors d'une ZUS occupent un emploi de cette catégorie.

Les écarts-types figurent entre parenthèses. Pour ce tableau, il s'agit des écarts-types empiriques calculés à partir d'une méthode de bootstrap. Le bootstrap consiste à simuler un grand nombre de fois l'échantillon (1000 fois ici) à partir d'un tirage avec remise depuis l'échantillon original.

Champ : jeunes sortis du système éducatif en 1998 résidant en ZUS (première colonne) ou dans une unité urbaine contenant une ZUS (deuxième colonne) ayant eu au moins un emploi au cours de leurs trois premières années de vie active.

Source : enquête Génération 98, première interrogation, Céreq. 


\section{L'évaluation des composantes des écarts salariaux}

T 'estimation d'un modèle d'accès aux différentes catégories d'emploi au moyen d'un modèle logit multinomial pour chacune des deux populations de jeunes (cf. tableaux 5 et 6 ) permet d'expliciter les déterminants individuels dans l'accès relatif aux différentes catégories d'emplois (celles figurant dans le tableau 4) pour les jeunes originaires de ZUS et les autres jeunes urbains. On peut donc, dans un premier temps, en comparant les deux tableaux, pointer d'éventuelles dif-

Tableau 5

L'accès aux différentes catégories d'emploi pour les jeunes résidant en ZUS Régression logistique multinomiale - Référence : ouvriers non qualifiés

\begin{tabular}{|c|c|c|c|c|c|c|}
\hline & $\begin{array}{c}\text { Ouvriers } \\
\text { non } \\
\text { qualifiés }\end{array}$ & $\begin{array}{l}\text { Ouvriers } \\
\text { qualifiés }\end{array}$ & $\begin{array}{c}\text { Employés } \\
\text { non } \\
\text { qualifiés }\end{array}$ & $\begin{array}{l}\text { Employés } \\
\text { qualifiés }\end{array}$ & $\begin{array}{l}\text { Profession } \\
\text { intermé- } \\
\text { diaire }\end{array}$ & Cadres \\
\hline Constante & - & $\begin{array}{l}-1,492^{* * *} \\
(0,240)\end{array}$ & $\begin{array}{l}0,464^{* *} \\
(0,190)\end{array}$ & $\begin{array}{l}-0,789^{* * \star} \\
(0,241)\end{array}$ & $\begin{array}{l}-1,817^{\star \star *} \\
(0,259)\end{array}$ & $\begin{array}{l}-5,846^{\star \star *} \\
(0,799)\end{array}$ \\
\hline $\begin{array}{l}\text { Femme } \\
\text { Homme }\end{array}$ & - & $\begin{array}{c}\text { Réf. } \\
0,568^{\star \star \star} \\
(0,193)\end{array}$ & $\begin{array}{c}\text { Réf. } \\
-1,636^{\star \star *} \\
(0,151)\end{array}$ & $\begin{array}{c}\text { Réf. } \\
-1,303^{\star \star \star} \\
(0,176)\end{array}$ & $\begin{array}{c}\text { Réf. } \\
-0,748^{\star \star \star} \\
(0,162)\end{array}$ & $\begin{array}{c}\text { Réf. } \\
-0,161^{\text {** }} \\
(0,242)\end{array}$ \\
\hline $\begin{array}{l}\text { Diplôme } \\
\text { Sans diplôme } \\
\text { CAP-BEP }\end{array}$ & - & $\begin{array}{l}\text { Réf. } \\
0,517^{\star \star \star} \\
(0,157)\end{array}$ & $\begin{array}{c}\text { Réf. } \\
0,252 \\
(0,162)\end{array}$ & $\begin{array}{c}\text { Réf. } \\
0,394^{*} \\
(0,227)\end{array}$ & $\begin{array}{c}\text { Réf. } \\
0,666^{\star \star} \\
(0,250)\end{array}$ & $\begin{array}{c}\text { Réf. } \\
-0,267 \\
(1,230)\end{array}$ \\
\hline Baccalauréat & - & $\begin{array}{c}0,024 \\
(0,212)\end{array}$ & $\begin{array}{c}0,284 \\
(0,184)\end{array}$ & $\begin{array}{l}1,261^{\star \star *} \\
(0,222)\end{array}$ & $\begin{array}{l}1,902^{\star \star *} \\
(0,234)\end{array}$ & $\begin{array}{r}1,587^{*} \\
(0,876)\end{array}$ \\
\hline Baccalauréat + 2 (BTS/DUT) & - & $\begin{array}{c}0,266 \\
(0,325)\end{array}$ & $\begin{array}{c}0,169 \\
(0,293)\end{array}$ & $\begin{array}{l}2,159^{* \star *} \\
(0,283)\end{array}$ & $\begin{array}{l}3,714^{\star \star \star} \\
(0,273)\end{array}$ & $\begin{array}{l}3,951^{* * *} \\
(0,789)\end{array}$ \\
\hline Baccalauréat + 3 & - & $\begin{array}{r}-0,112 \\
(0,819)\end{array}$ & $\begin{array}{l}0,753 \\
(0,480)\end{array}$ & $\begin{array}{l}1,726^{\star \star *} \\
(0,511)\end{array}$ & $\begin{array}{l}3,752^{\star \star \star} \\
(0,452)\end{array}$ & $\begin{array}{l}5,722^{\star \star *} \\
(0,863)\end{array}$ \\
\hline Baccalauréat +4 et plus & - & $\begin{array}{r}-1,290 \\
(1,059)\end{array}$ & $\begin{array}{c}0,428 \\
(0,432)\end{array}$ & $\begin{array}{l}2,501^{\star * *} \\
(0,405)\end{array}$ & $\begin{array}{l}3,842^{* \star *} \\
(0,391)\end{array}$ & $\begin{array}{l}7,483^{\star \star *} \\
(0,796)\end{array}$ \\
\hline $\begin{array}{l}\text { Autres spécialités } \\
\text { Spécialité industrielle }\end{array}$ & - & 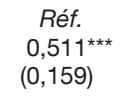 & $\begin{array}{c}\text { Réf. } \\
-0,875^{\star \star \star} \\
(0,162)\end{array}$ & $\begin{array}{c}\text { Réf. } \\
-1,144^{\star \star *} \\
(0,212)\end{array}$ & $\begin{array}{c}\text { Réf. } \\
-0,836^{\star \star \star} \\
(0,180)\end{array}$ & $\begin{array}{c}\text { Réf. } \\
0,123 \\
(0,310)\end{array}$ \\
\hline $\begin{array}{l}\text { Profession du père } \\
\text { Père ouvrier, employé, autres } \\
\text { Père artisan, commerçant } \\
\text { Père profession intermédiaire } \\
\text { Père cadre }\end{array}$ & - & $\begin{array}{c}\text { Réf. } \\
-0,1066 \\
(0,283) \\
-0,1474 \\
(0,160) \\
0,1064 \\
(0,348)\end{array}$ & $\begin{array}{c}\text { Réf. } \\
0,627^{\star \star} \\
(0,253) \\
0,207 \\
(0,149) \\
0,166 \\
(0,328)\end{array}$ & $\begin{array}{c}\text { Réf. } \\
-0,0201 \\
(0,327) \\
0,0635 \\
(0,175) \\
0,0406 \\
(0,344)\end{array}$ & $\begin{array}{c}\text { Réf. } \\
0,657^{\star *} \\
(0,275) \\
0,181 \\
(0,166) \\
0,661^{\star *} \\
(0,298)\end{array}$ & $\begin{array}{c}\text { Réf. } \\
0,762^{\star} \\
(0,429) \\
0,447 \\
(0,289) \\
1,115^{\star \star \star} \\
(0,382)\end{array}$ \\
\hline $\begin{array}{l}\text { Lieu de naissance des parents } \\
\text { Les deux parents nés en France ou de } \\
\text { nationalité française } \\
\text { Un des } 2 \text { parents ou les } 2 \text { nés hors de } \\
\text { France }\end{array}$ & - & $\begin{array}{c}\text { Réf. } \\
-0,1557 \\
(0,146)\end{array}$ & $\begin{array}{c}\text { Réf. } \\
-0,1760 \\
(0,140)\end{array}$ & $\begin{array}{c}\text { Réf. } \\
-0,1056 \\
(0,165)\end{array}$ & $\begin{array}{c}\text { Réf. } \\
0,0045 \\
(0,156)\end{array}$ & $\begin{array}{c}\text { Réf. } \\
0,2297 \\
(0,264)\end{array}$ \\
\hline $\begin{array}{l}\text { Ratio (Cadres + Professions intermé- } \\
\text { diaires) / (Employés + Ouvriers) }\end{array}$ & - & $\begin{array}{l}0,0005 \\
(0,003)\end{array}$ & $\begin{array}{l}0,0048 \\
(0,003)\end{array}$ & $\begin{array}{c}0,0051 \\
(0,003)\end{array}$ & $\begin{array}{l}0,008^{\star \star *} \\
(0,003)\end{array}$ & $\begin{array}{l}0,014^{\star * *} \\
(0,004)\end{array}$ \\
\hline $\begin{array}{l}\text { Résider hors île-de-France à la fin des } \\
\text { études } \\
\text { Résider en Île-de-France à la fin des } \\
\text { études }\end{array}$ & - & $\begin{array}{c}\text { Réf. } \\
0,1509 \\
(0,179)\end{array}$ & $\begin{array}{l}\text { Réf. } \\
0,3099^{*} \\
(0,168)\end{array}$ & $\begin{array}{l}\text { Réf. } \\
0,5632^{\star \star *} \\
(0,190)\end{array}$ & $\begin{array}{l}\text { Réf. } \\
0,683^{\star \star *} \\
(0,178)\end{array}$ & $\begin{array}{c}\text { Réf. } \\
0,481^{*} \\
(0,282)\end{array}$ \\
\hline $\begin{array}{l}\text { Log-Vraisemblance } \\
\text { Nombre d'observations } \\
\text { Statistique } L R \\
P \text {-Value } \\
\text { Pseudo } R^{2}\end{array}$ & & & $\begin{array}{r}-3 \\
2 \\
2 \\
0 \\
0\end{array}$ & $\begin{array}{l}2,6 \\
5 \\
13 \\
0 \\
10\end{array}$ & & \\
\hline
\end{tabular}

Lecture : régression logistique multinomiale. Les seuils de significativité sont respectivement égaux à $\left.1 \%\left(^{* * *}\right), 5 \%{ }^{* *}\right)$ et $10 \%\left(^{*}\right)$. La modalité d'accès à l'emploi de référence est celle qui consiste à devenir ouvrier non qualifié. Les caractéristiques individuelles de référence sont les suivantes : femme sans diplôme, ayant suivi une formation générale ou tertiaire, de père ouvrier ou employé, ayant les deux parents nés en France ou de nationalité française, résidant hors d'Île-de-France à la fin des études.

Relativement aux caractéristiques de ceux qui deviennent ouvriers non qualifiés, les jeunes résidant en ZUS qui deviennent employés qualifiés sont moins souvent des hommes, le coefficient associé à cette variable étant statistiquement significativement positif, au seuil de $1 \%$ Champ : jeunes sortis du système éducatif en 1998 résidant en ZUS, ayant eu au moins un emploi au cours de leurs trois premières années de vie active.

Source : enquête Génération 98, première interrogation, Céreq. 
férences dans l'effet de certaines caractéristiques (par rapport à la catégorie de référence).

\section{Pour les jeunes issus de ZUS, pas ou peu de barrières spécifiques dans l'accès aux différentes catégories d'emplois}

En premier lieu, l'accès aux différents groupes d'emplois est structuré par le capital humain des jeunes. En particulier, l'accès aux catégories de cadre lors de la première embauche est conditionné par la possession d'un diplôme au moins égal à la licence. De plus, le rôle des diplômes apparaît similaire dans l'allocation aux catégories d'emploi au sein des deux populations. Les effets liés à la spécialité de formation ne se différencient que sur l'accès aux emplois de cadres : alors que les spécialités à dominante industrielle ou scientifique réduisent la probabilité d'obte-

Tableau 6

L'accès aux différentes catégories d'emploi pour les jeunes résidant hors d'une ZUS Régression logistique multinomiale - Référence : ouvriers non qualifiés

\begin{tabular}{|c|c|c|c|c|c|c|}
\hline & $\begin{array}{l}\text { Ouvriers } \\
\text { non } \\
\text { qualifiés }\end{array}$ & $\begin{array}{l}\text { Ouvriers } \\
\text { qualifiés }\end{array}$ & $\begin{array}{l}\text { Employés } \\
\text { non } \\
\text { qualifiés }\end{array}$ & $\begin{array}{l}\text { Employés } \\
\text { qualifiés }\end{array}$ & $\begin{array}{l}\text { Professions } \\
\text { intermé- } \\
\text { diaires }\end{array}$ & Cadres \\
\hline Constante & - & $\begin{array}{l}-1,563^{\star \star \star} \\
(0,111)\end{array}$ & $\begin{array}{l}0,611^{\star \star \star} \\
(0,089)\end{array}$ & $\begin{array}{c}-0,971^{\star \star *} \\
(0,118)\end{array}$ & $\begin{array}{l}-1,177^{\star \star \star} \\
(0,111)\end{array}$ & $\begin{array}{l}-5,109^{* * *} \\
(0,369)\end{array}$ \\
\hline $\begin{array}{l}\text { Femme } \\
\text { Homme }\end{array}$ & - & $\begin{array}{l}\text { Réf. } \\
0,475^{\star \star \star} \\
(0,090)\end{array}$ & $\begin{array}{l}\text { Réf. } \\
-1,591^{\star \star \star} \\
(0,067)\end{array}$ & $\begin{array}{l}\text { Réf. } \\
-1,421^{\star \star *} \\
(0,071)\end{array}$ & $\begin{array}{l}\text { Réf. } \\
-0,946^{\star \star \star} \\
(0,066)\end{array}$ & $\begin{array}{l}\text { Réf. } \\
-0,369^{\star \star \star} \\
(0,077)\end{array}$ \\
\hline $\begin{array}{l}\text { Diplôme } \\
\text { Sans diplôme } \\
\text { CAP-BEP } \\
\text { Baccalauréat } \\
\text { Baccalauréat + } 2 \text { (BTS/DUT) } \\
\text { Baccalauréat + } 3 \\
\text { Baccalauréat }+4 \text { et plus }\end{array}$ & $\begin{array}{l}- \\
- \\
- \\
- \\
-\end{array}$ & $\begin{array}{c}\text { Réf. } \\
0,556^{\star \star \star} \\
(0,076) \\
0,327^{\star \star \star} \\
(0,087) \\
0,148 \\
(0,116) \\
0,497^{\star \star} \\
(0,240) \\
0,331^{\star} \\
(0,197)\end{array}$ & $\begin{array}{l}\text { Réf. } \\
0,216^{\star \star \star} \\
(0,078) \\
0,263^{\star \star \star} \\
(0,082) \\
0,231^{\star \star} \\
(0,102) \\
0,849^{\star \star \star} \\
(0,173) \\
0,678^{\star \star \star} \\
(0,151)\end{array}$ & $\begin{array}{c}\text { Réf. } \\
0,981^{\star \star \star} \\
(0,113) \\
1,561^{\star \star \star \star} \\
(0,111) \\
2,336^{\star \star \star} \\
(0,120) \\
2,425^{\star \star \star} \\
(0,186) \\
2,987^{\star \star \star} \\
(0,159)\end{array}$ & $\begin{array}{c}\text { Réf. } \\
0,209^{\star \star \star} \\
(0,116) \\
1,645^{\star \star \star} \\
(0,103) \\
3,428^{\star \star \star} \\
(0,108) \\
3,575^{\star \star \star} \\
(0,173) \\
3,926^{\star \star \star} \\
(0,149)\end{array}$ & $\begin{array}{c}\text { Réf. } \\
0,291 \\
(0,468) \\
1,922^{\star \star \star} \\
(0,388) \\
3,735^{\star \star \star} \\
(0,371) \\
6,093^{\star \star \star} \\
(0,390) \\
7,952^{\star \star \star} \\
(0,376)\end{array}$ \\
\hline $\begin{array}{l}\text { Autres spécialités } \\
\text { Spécialité industrielle }\end{array}$ & - & $\begin{array}{l}\text { Réf. } \\
0,416^{\star \star \star} \\
(0,070)\end{array}$ & $\begin{array}{l}\text { Réf. } \\
-1,178^{\star \star \star} \\
(0,070)\end{array}$ & $\begin{array}{l}\text { Réf. } \\
-1,490^{\star \star *} \\
(0,083)\end{array}$ & $\begin{array}{l}\text { Réf. } \\
-0,941^{\star * *} \\
(0,067)\end{array}$ & $\begin{array}{c}\text { Réf. } \\
-0,339^{\star \star \star} \\
(0,088)\end{array}$ \\
\hline $\begin{array}{l}\text { Profession du père } \\
\text { Père ouvrier, employé, autres } \\
\text { Père artisan, commerçant } \\
\text { Père profession intermédiaire } \\
\text { Père cadre }\end{array}$ & - & $\begin{array}{c}\text { Réf. } \\
0,284^{\star \star \star} \\
(0,103) \\
0,0306 \\
(0,068) \\
0,1353 \\
(0,108)\end{array}$ & $\begin{array}{c}\text { Réf. } \\
0,358^{\star \star \star} \\
(0,100) \\
0,239^{\star \star \star} \\
(0,066) \\
0,469^{\star \star \star} \\
(0,098)\end{array}$ & $\begin{array}{c}\text { Réf. } \\
0,390^{\star \star \star} \\
(0,109) \\
0,333^{\star \star \star} \\
(0,072) \\
0,606^{\star \star \star} \\
(0,100)\end{array}$ & $\begin{array}{c}\text { Réf. } \\
0,472^{\star \star \star} \\
(0,099) \\
0,2188^{\star \star \star} \\
(0,067) \\
0,6413^{\star \star \star} \\
(0,092)\end{array}$ & $\begin{array}{c}\text { Réf. } \\
0,439^{\star \star \star} \\
(0,124) \\
0,095 \\
(0,091) \\
0,938^{\star \star \star} \\
(0,107)\end{array}$ \\
\hline $\begin{array}{l}\text { Lieu de naissance des parents } \\
\text { Les deux parents nés en France ou de } \\
\text { nationalité française } \\
\text { Un des } 2 \text { parents ou les } 2 \text { nés hors de } \\
\text { France }\end{array}$ & - & $\begin{array}{c}\text { Réf. } \\
-0,130^{\star} \\
(0,070)\end{array}$ & $\begin{array}{c}\text { Réf. } \\
0,0656 \\
(0,067)\end{array}$ & $\begin{array}{c}\text { Réf. } \\
-0,185^{\star *} \\
(0,076)\end{array}$ & $\begin{array}{c}\text { Réf. } \\
-0,150^{\star *} \\
(0,067)\end{array}$ & $\begin{array}{c}\text { Réf. } \\
-0,195^{\star \star} \\
(0,093)\end{array}$ \\
\hline $\begin{array}{l}\text { Ratio (Cadres + Professions intermé- } \\
\text { diaires) / (Employés + Ouvriers) }\end{array}$ & - & $\begin{array}{c}0,0021^{\star * *} \\
(0,001)\end{array}$ & $\begin{array}{c}0,0037^{\star \star \star} \\
(0,001)\end{array}$ & $\begin{array}{c}0,004^{\star \star *} \\
(0,001)\end{array}$ & $\begin{array}{c}0,0047^{\star \star \star *} \\
(0,001)\end{array}$ & $\begin{array}{c}0,0076^{\star \star *} \\
(0,001)\end{array}$ \\
\hline $\begin{array}{l}\text { Résider hors île-de-France à la fin des } \\
\text { études } \\
\text { Résider en Île-de-France à la fin des } \\
\text { études }\end{array}$ & - & $\begin{array}{c}\text { Réf. } \\
0,169^{\star \star \star} \\
(0,078)\end{array}$ & $\begin{array}{c}\text { Réf. } \\
0,387^{\star \star \star} \\
(0,073)\end{array}$ & $\begin{array}{c}\text { Réf. } \\
0,612^{\star \star \star} \\
(0,078)\end{array}$ & $\begin{array}{c}\text { Réf. } \\
0,535^{\star \star \star} \\
(0,073)\end{array}$ & $\begin{array}{c}\text { Réf. } \\
0,614^{\star \star \star} \\
(0,087)\end{array}$ \\
\hline $\begin{array}{l}\text { Log-Vraisemblance } \\
\text { Nombre d'observations } \\
\text { Statistique LR } \\
\text { P-Value } \\
\text { Pseudo R2 }\end{array}$ & & & $\begin{array}{r}-2 \\
2 \\
2\end{array}$ & $\begin{array}{l}149 \\
192 \\
27 \\
00 \\
84\end{array}$ & & \\
\hline
\end{tabular}

Lecture : régression logistique multinomiale. Les seuils de significativité sont respectivement égaux à $1 \%\left({ }^{* * *}\right), 5 \%\left(^{* *}\right)$ et $10 \%\left(^{*}\right)$. La modalité d'accès à l'emploi de référence est celle qui consiste à devenir ouvrier non qualifié. Les caractéristiques individuelles de référence sont les suivantes : femme sans diplôme, ayant suivi une formation générale ou tertiaire, de père ouvrier ou employé, ayant les deux parents nés en France ou de nationalité française, résidant hors d'Île-de-France à la fin des études.

Relativement aux caractéristiques de ceux qui deviennent ouvriers non qualifiés, les jeunes résidant hors ZUS qui deviennent employés qualifiés sont moins souvent des hommes, le coefficient associé à cette variable étant statistiquement significativement négatif, au seuil de $1 \%$. Champ : jeunes sortis du système éducatif en 1998 résidant hors d'une ZUS mais dans une unité urbaine contenant une ZUS, ayant eu au moins un emploi au cours de leurs trois premières années de vie active.

Source : enquête Génération 98, première interrogation, Céreq. 
nir un premier emploi de cadre chez les jeunes urbains, cette spécificité disparaît pour les jeunes issus de ZUS.

L'influence du sexe est également marquée : les jeunes femmes ont beaucoup plus de chances d'accéder aux fonctions tertiaires et donc aux professions d'employés et, dans une moindre mesure, aux professions intermédiaires et aux postes de cadre (10). Là encore, l'impact du sexe est assez proche entre les jeunes issus de ZUS et les autres, sauf dans l'accès aux emplois de cadres ; il semble que les jeunes filles soient moins avantagées lorsqu'elles sont issues de ZUS, l'intensité du coefficient associé au genre faisant plus que doubler (il passe de 0,161 à $0,369)$ entre les tableaux 5 et 6.

Par ailleurs, le capital social des parents, approché ici par la profession du père, s'avère très discriminant dans l'accès aux professions les plus qualifiées pour les deux groupes de jeunes. Ainsi, avoir un père cadre augmente très fortement, « toutes choses égales par ailleurs », les chances d'accéder à un emploi de cadre. En revanche, l'origine géographique des parents semble peu intervenir pour expliquer l'accès aux différentes catégories d'emplois des jeunes résidant en ZUS alors que pour les autres jeunes urbains, elle freine l'accès aux professions les plus qualifiées lorsque les parents sont nés hors de France.

Enfin, les contextes territoriaux influencent également l'accès aux différentes catégories d'emploi. Que l'on réside en ZUS ou non, habiter en Île-de-France est un facteur favorable pour l'accès aux emplois de cadre et de professions intermédiaires. Mais la probabilité d'occuper les emplois de catégorie élevée tient également, pour les jeunes issus de ZUS comme pour les autres, aux effets de voisinage mesurés ici par la structure des emplois des habitants du quartier : plus les emplois de cadres et professions intermédiaires s'y trouvent représentés (relativement aux autres catégories) et plus les jeunes, « toutes choses égales par ailleurs », peuvent eux-mêmes y avoir accès. Les jeunes issus de ZUS semblent plus sensibles à la structure locale des emplois que les autres, soit parce qu'ils sont moins mobiles, soit parce que leurs informations sur les offres d'emploi disponibles sont plus limitées à leur environnement immédiat, soit parce qu'ils sont moins discriminés lorsqu'ils accèdent aux emplois qualifiés dans leur lieu de résidence.

En fin de compte, si certains déterminants se distinguent d'une population à l'autre dans l'allocation aux catégories d'emploi, une fois considérés globalement ces facteurs conduisent à des modèles d'allocation très similaires. Cela laisse supposer qu'il n'existe pas ou peu, concernant les jeunes issus de ZUS, de barrières spécifiques dans l'accès aux différentes catégories d'emplois, impliquant a priori une faible valeur explicative de la discrimination dans l'allocation aux emplois sur le différentiel de salaires observé entre les deux groupes de jeunes. Pour preuve, la structure hypothétique (11) des emplois des jeunes issus de ZUS, s'ils résidaient dans un quartier hors ZUS d'une agglomération contenant une ZUS, est très proche de la structure des emplois réellement observée pour cette population (cf. tableau 4, dernière colonne). Ainsi, si les jeunes originaires de ZUS étaient traités par les employeurs dans l'accès aux différents emplois comme le sont les autres jeunes, la structure de leurs emplois serait globalement inchangée.

\section{Des écarts de salaire entre les deux populations davantage liés à des différences de capacité d'accès aux différentes catégories d'emploi qu'à des différences de rémunération dans chaque catégorie}

Avant de développer la méthode de décomposition de l'écart salarial entre les deux populations proposée par Brown, Moon et Zoloth (1980), on peut d'abord vérifier que les niveaux de salaires dépendent de la catégorie d'emploi occupée (12). L'estimation des équations de salaires pour les deux populations confirme l'influence de la catégorie d'emploi sur le niveau de rémunération (cf. annexe 2, tableau A) (13). Concernant les autres facteurs explicatifs des niveaux de salaire, le niveau de capital humain des jeunes (leur diplôme le plus élevé, leur spé-

10. II faut lire cet effet par rapport à l'emploi de référence qu'est la catégorie " ouvrier non qualifié ", les jeunes filles ayant moins de chances de se trouver dans ce type d'emploi.

11. Si les facteurs influençant l'accès aux emplois (niveau d'éducation, origine sociale, ...) jouaient de la même manière pour les deux populations.

12. Rappelons qu'une des limites de la décomposition est de ne pas tenir compte d'un effet de sélection éventuel dans l'accès à l'emploi qui peut s'expliquer notamment par des caractéristiques individuelles non observées. Nous avons cependant vérifié que la prise en compte de ce type de biais n'affectait pas globalement les composantes expliquées et non expliquées de l'écart de salaire à partir de décompositions intégrant un terme de sélectivité spécifique dans l'explication de l'écart salarial (Neuman et Oaxaca, 1998), mais ne tenant plus compte de l'affectation à différents types d'emploi.

13. Les deux équations de salaires où les catégories d'emplo sont introduites en variables explicatives figurent en annexe 2. Par la suite, pour la décomposition de l'écart salarial, une équation de salaire par catégorie d'emploi et par groupe de jeunes est estimée. 
cialité de formation, les stages durant les études) et le contexte territorial expliquent une part importante de la rémunération, quel que soit le type de quartier d'origine. Un test de Chow permettant de mesurer l'égalité des coefficients pour chaque modalité des variables introduites dans les deux fonctions de gains montre que les déterminants du salaire sont très proches pour les deux sous-populations. Seuls diffèrent les coefficients associés à la spécialité de formation, au temps de travail et à l'environnement socio-économique du quartier, pris en compte ici par la variable « nombre de cadres et de professions intermédiaires sur nombre d'ouvriers et d'employés ». Comme pour l'accès aux différentes catégories d'emploi, cet effet de voisinage montre que les jeunes issus de ZUS sont plus sensibles que les autres à une amélioration de la structure des emplois des habitants de leur quartier.

Passons maintenant aux résultats relatifs à la décomposition de l'écart de salaire entre les jeunes issus de ZUS et les autres jeunes urbains. Globalement, le salaire moyen des jeunes qui ne résident pas en ZUS à la fin de leur formation initiale en 1998 est de 13,0\% supérieur à celui des jeunes habitant une ZUS à cette date. La décomposition de cet écart salarial (cf. tableau 7) montre que plus de $90 \%$ de celui-ci est lié à des différences de caractéristiques individuelles explicatives de l'accès à l'emploi ou de la rémunération. Autrement dit, si les jeunes des deux populations avaient les mêmes caractéristiques individuelles (même niveau de diplôme, même origine sociale, etc.) et le même type de voisinage, l'écart salarial qui subsisterait, lié à des différences de rendement de ces caractéristiques dans l'accès aux emplois ou dans la rémunération, serait alors très faible. Les difficultés des jeunes résidant en ZUS - relativement aux autres jeunes urbains - pour accéder aux emplois les mieux rémunérés et les plus qualifiés apparaissent donc principalement liées aux caractéristiques déterminées avant leur entrée sur le marché du travail, mais peu au rendement de

Tableau 7

La décomposition de l'écart de salaire moyen entre les jeunes résidant en ZUS et les autres, à l'embauche du premier emploi

\begin{tabular}{|c|c|}
\hline & Écarts des gains \\
\hline Écart estimé de salaires mensuels en log et en \% & $\begin{array}{l}0,123^{\star \star \star}(\text { soit } 13,04 \%) \\
(0,008)\end{array}$ \\
\hline \multicolumn{2}{|c|}{ Écart de salaires lié aux différences internes à chaque catégorie d'emploi (en log) } \\
\hline Écart (« intra-catégorie ») & $\begin{array}{l}0,037^{\star \star *} \\
(0,008)\end{array}$ \\
\hline (1) expliqué par les différences de caractéristiques & $\begin{array}{l}0,032^{\star * *} \\
(0,005)\end{array}$ \\
\hline $\begin{array}{l}\text { (2) avantage des « hors ZUS » non expliqué par les différen- } \\
\text { ces de caractéristiques }\end{array}$ & $\begin{array}{l}0,001 \\
(0,001)\end{array}$ \\
\hline $\begin{array}{l}\text { (3) désavantage des « ZUS » non expliqué par les différences } \\
\text { de caractéristiques }\end{array}$ & $\begin{array}{c}0,004 \\
(0,005)\end{array}$ \\
\hline \multicolumn{2}{|c|}{$\begin{array}{l}\text { Écart de salaires lié au fait que les individus des deux populations n'ont pas les mêmes emplois } \\
\text { (en log) }\end{array}$} \\
\hline Écart (« inter-catégorie ») & $\begin{array}{l}0,086^{\star \star *} \\
(0,004)\end{array}$ \\
\hline (4) expliqué par les différences de caractéristiques & $\begin{array}{l}0,090^{* \star *} \\
(0,003)\end{array}$ \\
\hline (5) non expliqué par les différences de caractéristiques & $\begin{array}{c}-0,004 \\
(0,003)\end{array}$ \\
\hline $\begin{array}{l}\text { Total expliqué par les différences de caractéristiques } \\
((1)+(4))\end{array}$ & $\begin{array}{l}0,122^{\star \star \star} \\
(0,006)\end{array}$ \\
\hline $\begin{array}{l}\text { Total non expliqué par les différences de caractéristiques } \\
((2)+(3)+(5))\end{array}$ & $\begin{array}{c}0,001 \\
(0,007)\end{array}$ \\
\hline
\end{tabular}

Lecture : l'écart de salaire au premier emploi entre les jeunes habitant dans des ZUS au moment de leur sortie du système éducatif et des jeunes ne résidant pas dans des ZUS mais habitant dans des unités urbaines comprenant des ZUS est de 0,123 en logarithme, soit $13,04 \%$ en faveur de ces derniers (ce pourcentage étant calculé en écrivant (exponentielle $(0,1226)$ )-1).

Cet écart provient pour plus des deux tiers des différences dans l'affectation des emplois (écart inter-catégorie) et pour un peu moins d'un tiers de différences de rémunération au sein de chaque emploi (écart intra-catégorie).

Les écarts types figurent entre parenthèses. II s'agit des écarts-types empiriques calculés à partir d'une méthode de bootstrap. Le bootstrap consiste à simuler un grand nombre de fois l'échantillon (1000 fois ici) à partir d'un tirage avec remise depuis l'échantillon original. Les seuils de significativité sont respectivement égaux à $1 \%\left(^{(* *}\right), 5 \%\left(^{* *}\right)$ et $10 \%\left(^{*}\right)$.Les écarts-types figurent entre parenthèses.

Champ : jeunes sortis du système éducatif en 1998 résidant en ZUS ou dans une unité urbaine contenant une ZUS, ayant eu au moins un emploi au cours de leurs trois premières années de vie active.

Source : enquête Génération 98, première interrogation, Céreq. 
ces caractéristiques déterminé par le fonctionnement du marché du travail. En conséquence, si l'on interprète ces différences de rendement comme de la discrimination salariale produite par le marché du travail, nos résultats montrent que ce type de discrimination est faible. Cela ne signifie pas pour autant qu'il n'y ait pas d'autres sources de discrimination à l'encontre des jeunes issus de ZUS en amont du marché du travail susceptibles de contribuer aux différences de salaires. Ainsi, les jeunes issus de ZUS ont pu souffrir de discriminations (directes ou indirectes) dans leur cursus scolaire et leur orientation pour atteindre les plus hauts niveaux d'éducation du fait, par exemple, de leur environnement scolaire, social et des caractéristiques locales de l'offre de formation. Ces différences de « valorisation », non identifiables au travers des données mobilisées ici, se cristallisent alors dans nos résultats dans la part de l'écart de salaire attribuée aux caractéristiques individuelles, telles qu'elles sont observées à leur entrée sur le marché du travail.

Revenons maintenant à la part que tient l'allocation aux catégories d'emploi dans l'explication de l'écart de rémunération. La décomposition indique que les différences salariales entre les deux populations sont davantage liées à des différences d'accès aux différentes catégories d'emploi qu'à des différences de rémunération au sein de chaque catégorie. La composante inter-catégorie de l'écart salarial (70\%) est plus de deux fois supérieure à la composante intra-catégorie (14). Autrement dit, si les jeunes issus de ZUS pouvaient accéder aux catégories de cadres et de professions intermédiaires dans les mêmes proportions que jeunes issus d'autres quartiers urbains, les différences de salaires entre les deux populations seraient réduites des deux tiers. Comme souligné précédemment, ce constat est essentiellement le fruit de différences dans les caractéristiques individuelles des jeunes de ZUS relativement aux autres, caractéristiques qui leur permettent d'accéder plus difficilement aux emplois les plus élevés de la hiérarchie sociale, qui sont également les mieux rétribués.

\section{Les effets qui ne relèvent pas de différences de caractéristiques sont peu significatifs}

Concernant l'écart salarial inexpliqué par les caractéristiques individuelles, les composantes inter- et intra-catégorie sont faibles et de signe opposé. De plus, les écarts-types qui y sont associés montrent qu'aucun effet n'est significatif. La composante intra-catégorie, légèrement plus forte en valeur absolue, nous indique qu'à catégorie d'emploi identique et pour les mêmes attributs individuels, les jeunes issus de ZUS semblent un peu moins payés que les autres jeunes urbains. Cet effet n'est cependant pas statistiquement différent de zéro, ce qui conduit à relativiser une explication par exemple en termes de discrimination territoriale (15). Le constat est analogue pour la composante non expliquée inter-catégorie qui, d'un signe négatif, n'est pas significativement différente de zéro. Là encore, l'absence de significativité de l'effet ne permet pas d'établir l'existence d'une discrimination dans l'accès à l'emploi.

\section{Une méthode de décomposition pour chaque catégorie d'emploi}

L'analyse proposée précédemment tient compte de l'allocation des individus à différents types d'emplois. Il est également possible de proposer une décomposition pour chaque catégorie sociale selon la méthode d'Oaxaca-Ransom (1994). Cette dernière méthode ne tient pas compte de l'écart inter-catégorie, mais permet de préciser et d'expliquer au sein de chaque catégorie, les écarts de rémunération entre jeunes issus de ZUS et jeunes issus d'autres quartiers urbains. Les résultats (cf. tableau 8, première colonne) confirment l'existence d'écarts de salaire, notamment pour les professions les plus qualifiés : employés qualifiés, professions intermédiaires et cadres. Pour ces trois catégories, les écarts de salaires sont les plus élevés et significatifs au seuil de $5 \%$. Lorsque ces écarts sont décomposés en une partie liée aux caractéristiques individuelles (cf. tableau 8, colonne 2) et une partie non expliquée que l'on pourrait attribuer à de la discrimination ou à d'autres facteurs plus difficilement observables comme l'insuffisance d'information due à la faiblesse des réseaux sociaux (cf. tableau 8, colonne 3 ), les effets liés au second terme n'apparaissent pas négligeables pour les catégories où les écarts

14. Précisons que ce résultat est en partie dépendant de la nomenclature et de la hiérarchie des catégories d'emploi retenues. Une nomenclature plus fine risquerait d'aboutir à un partage de l'écart salarial inter / intra encore plus favorable à la composante inter-catégorie.

15. Outre l'hypothèse d'une discrimination, d'autres types d'explications auraient pu être données comme par exemple l'existence de différents recours aux modes de recherche d'emploi ou la présence de salaires de réserves moins élevés parce que les jeunes issus de ZUS anticipent une discrimination sur le marché du travail et/ou parce que leurs ressources financières ne leur permettent pas de supporter une durée de recherche trop élevée. 
salariaux sont les plus importants : ils représentent respectivement $53 \%, 24 \%$ et $59 \%$ des écarts de salaires des employés qualifiés, professions intermédiaires et cadres. Cependant, seul le terme non expliqué associé aux emplois non qualifiés est significatif. Les différences de caractéristiques individuelles expliquent toujours une part non négligeable des différences de salaires, part qui est notamment significative pour les employés qualifiés et pour les professions intermédiaires. Notons que cette seconde décomposition n'invalide pas les résultats de la précédente, beaucoup plus générale car portant sur l'ensemble des premiers emplois auxquels les jeunes ont pu accéder, mais la complète dans la mesure où elle apporte des enseignements pour des jeunes accédant à un même type d'emploi. On ne peut exclure l'hypothèse d'un effet lié à la discrimination pour les emplois les plus qualifiés où les écarts de salaires sont importants. Cependant, dans le cas des cadres notamment où l'effet n'est pas significatif, la taille limitée de l'échantillon pour les jeunes résidant en ZUS ne permet pas de conforter ou d'infirmer totalement cette hypothèse alors qu'il aurait été au contraire intéressant de décomposer cette catégorie en fonction par exemple du type d'employeur ou du secteur d'activité.

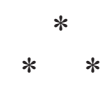

À partir de données sur l'insertion professionnelle des jeunes, cet article a étudié les écarts de rémunération entre jeunes issus de ZUS et jeunes issus d'autres quartiers urbains lors de leur accès au marché du travail. Nous nous sommes notamment intéressés à l'existence de barrières spécifiques dans l'accès à certaines catégories d'emplois, qui pénaliseraient les jeunes issus de ZUS. Nous avons montré que ces derniers ont effectivement plus de difficultés à accéder aux emplois les plus qualifiés et obtiennent des rémunérations moins élevées que les jeunes issus d'autres quartiers. De plus, l'écart de salaires au premier emploi entre les deux sous-populations, d'environ $13 \%$, s'explique principalement par l'accès plus difficile des jeunes issus de ZUS aux catégories d'emplois les mieux rémunérés. Cependant, le principal résultat de l'article est que la quasi-totalité de l'écart de salaire entre jeunes issus de ZUS et jeunes non issus de ZUS tient à des différences de caractéristiques individuelles liées notamment à leur capital humain ou aux caractéristiques socioéconomiques de leur voisinage. Autrement dit, si les jeunes issus de ZUS avaient les mêmes caractéristiques que les jeunes issus d'autres quartiers et résidaient dans des environnements socioéconomiques comparables, ils auraient les mêmes chances d'accès aux emplois les plus qualifiés et aux salaires les plus élevés. Ces résultats peuvent sans doute trouver leur origine dans de fortes inégalités en

\section{Tableau 8}

Décomposition d'Oaxaca - Ransom des écarts de salaire à l'embauche du premier emploi, selon la catégorie d'emploi

\begin{tabular}{|c|c|c|c|c|c|}
\hline & \multirow[b]{2}{*}{$\begin{array}{l}\text { Écart salarial } \\
\quad(e n \text { log) }\end{array}$} & \multicolumn{2}{|c|}{ Décomposition de l'écart salarial } & \multirow[b]{2}{*}{$\begin{array}{c}\text { Effectif } \\
\text { ZUS }\end{array}$} & \multirow[b]{2}{*}{$\begin{array}{c}\text { Effectif } \\
\text { hors ZUS }\end{array}$} \\
\hline & & $\begin{array}{l}\text { Écart expliqué par } \\
\text { les différences de } \\
\text { caractéristiques } \\
\text { individuelles }\end{array}$ & $\begin{array}{c}\text { Écart } \\
\text { non expliqué }\end{array}$ & & \\
\hline Ouvriers non qualifiés & $\begin{array}{c}0,027^{*} \\
(0,015)\end{array}$ & $\begin{array}{c}0,029 \\
(0,009)\end{array}$ & $\begin{array}{c}-0,002 \\
(0,011)\end{array}$ & 637 & 2902 \\
\hline Ouvriers qualifiés & $\begin{array}{c}0,018 \\
(0,019)\end{array}$ & $\begin{array}{c}0,009 \\
(0,009)\end{array}$ & $\begin{array}{c}0,009 \\
(0,017)\end{array}$ & 342 & 1953 \\
\hline Employés non qualifiés & $\begin{array}{c}0,008 \\
(0,018)\end{array}$ & $\begin{array}{c}0,009 \\
(0,011)\end{array}$ & $\begin{array}{c}-0,008 \\
(0,014)\end{array}$ & 502 & 3192 \\
\hline Employés qualifiés & $\begin{array}{c}0,077^{+*} \\
(0,020)\end{array}$ & $\begin{array}{c}0,036 \\
(0,013)\end{array}$ & $\begin{array}{c}0,041^{*} \\
(0,017)\end{array}$ & 307 & 2718 \\
\hline Professions intermédiaires & $\begin{array}{l}0,058^{* * *} \\
(0,018)\end{array}$ & $\begin{array}{l}0,044^{\text {w+t }} \\
(0,010)\end{array}$ & $\begin{array}{l}0,0137 \\
(0,013)\end{array}$ & 540 & 5613 \\
\hline Cadres & $\begin{array}{c}0,050^{* *} \\
(0,024)\end{array}$ & $\begin{array}{c}0,020 \\
(0,016)\end{array}$ & $\begin{array}{c}0,030 \\
(0,021)\end{array}$ & 197 & 4114 \\
\hline
\end{tabular}

Lecture : la première colonne présente pour chaque catégorie d'emploi, la différence d'écart salarial estimé (en logarithme) entre les jeunes urbains ne résidant pas dans une ZUS et les jeunes résidant dans une ZUS. Cet écart salarial, qui est de 0,027 en logarithme pour les ouvriers non qualifiés à l'avantage des jeunes ne résidant pas dans une ZUS, peut se décomposer en deux effets, un effet lié aux différences de caractéristiques individuelles (colonne 2), qui explique pour les ouvriers non qualifiés la totalité de l'écart salarial et un effet lié aux différences de traitement de ces caractéristiques (écart non expliqué, colonne 3), très faibles et non significatif pour les ouvriers non qualifiés.

Les seuils de significativité sont respectivement égaux à $1 \%\left(^{* *}\right), 5 \%\left(^{* *}\right)$ et $10 \%\left(^{*}\right)$.Les écarts-types figurent entre parenthèses.

Champ : jeunes sortis du système éducatif en 1998 résidant en ZUS ou dans une unité urbaine contenant une ZUS, ayant eu au moins un emploi au cours de leurs trois premières années de vie active.

Source : enquête Génération 98, première interrogation, Céreq. 
amont du marché du travail, inégalités qui sont à l'origine des différences d'insertion. Comme il apparaît que ces inégalités sont répercutées sans être réduites sur le marché du travail, une part importante de l'effort des pouvoirs publics devrait alors porter sur l'égalité des chances dans l'accès à l'éducation et à l'information sur les débouchés professionnels.

Ces résultats, qui concernent une décomposition concernant l'ensemble des emplois, des moins qualifiés au plus qualifiés, ne permettent pas de valider l'existence d'une forte discrimination salariale associée à la localisation résidentielle au détriment des habitants des ZUS. Les décompositions effectuées par catégories d'emploi conduisent cependant à quelques nuances qui peuvent s'avérer des pistes de réflexion pour des recherches futures. D'autres données, avec des échantillons plus grands ou d'autres méthodes centrées notamment sur l'étude des conditions de recrutement et de rémunération dans les emplois les plus qualifiés sont nécessaires pour progresser dans l'analyse. De plus, les catégories sociales que nous avons utilisées sont sans doute trop agrégées et regroupent des pratiques d'embauche et des politiques de rémunération très différentes selon le type d'entreprise ou le secteur d'activité.

\section{BIBLIOGRAPHIE}

Arrighi J.-J., Gasquet C. et Roux V. (2008), «Des mobilités résidentielles de début de carrière moins favorables aux femmes », Économie et Statistique, $\mathrm{n}^{\circ}$ 415-416, pp. 61-80.

Åslund O., Östh J. et Zenou Y. (2006), « How Important is Access to Jobs ? Old Question Improved Answer ", IZA Discussion Paper, $\mathrm{n}^{\circ} 2051$ (à paraître en 2010 dans Journal of Economic Geography).

Beaud S. (2002), $80 \%$ au bac... Et après ?, La Découverte, Paris.

Becker G.S. (1993 [1957]), The Economics of Discrimination, 2nd ed., University of Chicago Press.

Blinder A.S. (1973), «Wage Discrimination : Reduced Form and Structural Estimates », The Journal of Human Resources, vol. 8, n 4, pp. 436455.

Borjas G.J. (1992), « Ethnic Capital and Intergenerational Mobility », Quarterly Journal of Economics, vol. 107, $\mathrm{n}^{\circ}$ 1, pp. 123-150.

Borjas G.J. (1995), « Ethnicity, Neighbourhoods, and Human-Capital Externalities », American Economic Review, vol. 85, n 3, pp. 365-390.

Brown R.S., Moon M. et Zoloth B.S. (1980), « Incorporating Occupational Attainment in Studies of Male/Female Earnings Differentials », The Journal of Human Resources, vol. 15, $\mathrm{n}^{\circ} 1$, pp. 3-28
Brueckner J.K. et Zenou Y. (2003), « Space and Unemployment : The Labor-Market Effects of Spatial Mismatch », Journal of Labor Economics, vol. 21, n 1 , pp. 242-266.

Büchell F. et van Ham M. (2003), «Overeducation, Regional Labor Markets, and Spatial Flexibility », Journal of Urban Economics, vol. 53, $\mathrm{n}^{\circ} 3$, pp. 482-493.

Choffel P. et Delattre E. (2003), « Habiter un quartier défavorisé. Quels effets sur la durée de chômage? », Premières Synthèses, Dares, n 43.1, octobre.

Coulson N.E., Laing D. et Wang P. (2001), "Spatial Mismatch in Search Equilibrium " Journal of Labor Economics, vol. 19, $\mathrm{n}^{\circ} 4$, pp. 949-972.

Couppié T. et Gasquet C. (2006), « L'insertion professionnelle des jeunes originaires de ZUS », dans Observatoire National des Zones Urbaines Sensibles (2007), Rapport 2006, Editions de la DIV, Paris, pp. 210-232.

Couppié T. et Gasquet C. (2009), « Quartiers défavorisés : relégation pour certains jeunes, insertion sociale et professionnelle pour d'autres ", Céreq-Bref, $\mathrm{n}^{\circ} 261$, février.

De Stéphanis M., Mathey-Pierre C. et Dugué E. (2004), « De l'école à l'emploi : quels chemins dans les ZUS », Connaissance de l'Emploi, CEE, $\mathrm{n}^{\circ} 10$. 
Gobillon L. et Selod H. (2004), « Les déterminants spatiaux du chômage en Île-de-France », in Ségrégation Urbaine et Intégration Sociale, Fitoussi, Laurent and Maurice, éds., rapport du Conseil d'Analyse Économique, ${ }^{\circ}{ }^{\circ} 45$, La Documentation Française, Paris, pp. 171-187.

Gobillon L. et Selod H. (2007), « Les déterminants locaux du chômage en région parisienne », Économie et Prévision, $\mathrm{n}^{\circ}$ 180-181, pp. 19-38.

Gobillon L., Selod H. et Zenou Y. (2007), « The Mechanisms of Spatial Mismatch », Urban Studies, vol. 44, n 12, pp. 2401-2427.

Goux D. et Maurin É. (2005), « The Effect of Overcrowded Housing on Children's Performance at School », Journal of Public Economics, vol. 89, $\mathrm{n}^{\circ}$ 5-6, pp. 797-819.

Hellerstein J.K., Neumark D. et McInerney M. (2008), " Spatial Mismatch or Racial Mismatch ? ", Journal of Urban Economics, vol. $64, n^{\circ} 2$, pp. 464-479

Ilhanfeldt K. (1997), " Information on the Spatial Distribution of Job Opportunities Within Metropolitan Areas », Journal of Urban Economics, vol. 41, n 2, pp. 218-242.

Ihlanfeldt K.R. et Sjoquist D.L. (1998), « The Spatial Mismatch Hypothesis : a Review of Recent Studies and Their Implications for Welfare Reform », Housing Policy Debate, vol. 9, $\mathrm{n}^{\circ} 4$, pp. 849-892.

Ioannides Y.M. et Loury L.D. (2004), « Job Information Networks, Neighborhood Effects, and Inequality », Journal of Economic Literature, vol. 42, n 4, pp. 1056-1093.

Kain J. (1968), « Housing Segregation, Negro Employment, and Metropolitan Decentralization », Quarterly Journal of Economics, vol. 82, $\mathrm{n}^{\circ} 2$, pp. 175-197.

Le Toqueux J.-L. et Moreau J. (2002), « Les zones urbaines sensibles - Forte progression du chômage entre 1990 et 1999 », Insee Première, $\mathrm{n}^{\circ} 835$.

Loury L.D. (2006), « Some Contacts Are More Equal than Others : Informal Networks, Job Tenure, and Wages », Journal of Labor Economics, vol. 24, n 2 , pages 299-318.

Meng X. et Meurs D. (2001), « Différences de structure des emplois et écart salarial entre hom- mes et femmes en France », Économie et Prévision, $\mathrm{n}^{\circ} 148$, pp. 113-122.

Neuman S. et Oaxaca R.L. (1998), « Estimating Labour Market Discrimination with Selectivity Corrected Wage Equations : Methodological Considerations and an Illustration from Israel », CEPR Discussion Paper, $\mathrm{n}^{\circ} 1915$ (version revue et augmentée : (2004), « Wage Discrimination in the 1990s in Israel : Endowments, discrimination and Selectivity », IZA Discussion Paper, $n^{\circ}$ 1362).

Oaxaca R.L. (1973), « Male-Female Wage Differentials in Urban Labor Markets ", International Economic Review, vol. 14, $\mathrm{n}^{\circ} 3$, pp. 693-709.

Oaxaca R.L. et Ransom M.R. (1994), « On Discrimination and the Decomposition of Wage Differentials », Journal of Econometrics, vol. 61, $\mathrm{n}^{\circ} 1$, pp. 5-21.

Observatoire National des Zones Urbaines Sensibles (2004), Premier état des lieux de l'Observatoire National des Zones Urbaines Sensibles, Délégation Interministérielle à la Ville, Paris, (http ://www.ville.gouv.fr/IMG/pdf/observatoirezus_cle1d4fe5-1.pdf).

Observatoire National des Zones Urbaines Sensibles (2006), Rapport 2006, Éditions de la DIV, Paris (http ://www.ville.gouv.fr/pdf/editions/ observatoire-ZUS-rapport-2006.pdf).

Cuvrard F. et Rondeau M.-C. (2004), « Les inégalités de scolarisation : disparités sociales, et/ou territoriales », dans Ségrégation Urbaine et Intégration Sociale, J.-P. Fitoussi, É. Laurent et J. Maurice (éds.), rapport du Conseil d'Analyse Économique, $\mathrm{n}^{\circ} 45$, La Documentation Française, Paris, pp. 189-218.

Ortega J. (2000), « Pareto-Improving Immigration in an Economy with Equilibrium Unemployment », Economic Journal, vol. 110, n 460, pp. 92-112.

Pan Ké Shon J.-L (2007), « État des lieux des quartiers sensibles », Informations sociales, $\mathrm{n}^{\circ} 141$, Cnaf.

Pan Ké Shon J.-L (2009), « Ségrégation ethnique et ségrégation sociale en quartiers sensibles. L'apport des mobilités résidentielles ", Revue française de Sociologie, vol. 50, n 3, pp. 451-487.

Phelps E. (1972), « The Statistical Theory of Racism and Sexism ", American Economic Review, vol. 62, n 4, pp. 659-661. 
Raphael S. (1998), « The Spatial Mismatch Hypothesis and Black Youth Joblessness : Evidence From the San Francisco Bay Area ", Journal of Urban Economics, vol. 43, n 1, pp. 79-111.

Silberman R. et Fournier I. (1999), « Les enfants d'immigrés sur le marché du travail. Les mécanismes d'une discrimination sélective », FormationEmploi, n $^{\circ}$ 65, janvier-mars 1999, pp. 31-54.

Smith A.E. et Zenou Y. (2003), « A discrete-time stochastic model of job matching ", Review of Economic Dynamics, vol. 6, n 1, pp. 54-79.
Turner S.C. (1997), « Barriers to a Better Break : Employer Discrimination and Spatial Mismatch in Metropolitan Detroit », Journal of Urban Affairs, vol. $19, \mathrm{n}^{\circ} 2$, pp. 123-141.

Zenou Y. (2002), " How do Firms Redline Workers? » Journal of Urban Economics, vol. 52, $\mathrm{n}^{\circ} 3$, pp. 391-408. 


\section{DE LA DÉCOMPOSITION D'OAXACA-BLINDER-RANSOM À CELLE DE BROWN, MOON ET ZOLOTH}

\section{Le principe général des décompositions des écarts de salaire d'Oaxaca et de Blinder}

L'objectif des décompositions de l'écart de salaire existant entre deux groupes d'individus est de distinguer dans cet écart ce qui tient à des différences de caractéristiques entre les deux groupes (par exemple le salaire est plus élevé quand la qualification est supérieure) de ce qui provient d'une différence de valorisation de ces caractéristiques (par exemple, s'il y a discrimination salariale à l'encontre de l'un des deux groupes, telle qualification sera moins valorisée pour ce groupe que la « normale »).

L'exercice standard de décomposition repose sur l'estimation d'équations de salaire de la forme suivante :

$\operatorname{Ln} W_{i}^{\prime}=\beta^{\prime} X_{i}^{\prime}+u_{i}^{\prime}$

où :

$L n W_{i}$ est le logarithme du salaire individuel mensuel ; $x_{i}$ est un ensemble de caractéristiques relatives à l'individu $i$ et $u_{i}$ est le terme d'erreur.

L'indice / correspond aux groupes d'appartenance des individus (hommes et femmes ou nationaux et étrangers dans les nombreux travaux sur les discriminations salariales), ici les individus résidant dans une ZUS à la fin de leurs études ("ZUS ") et ceux résidant hors d'une ZUS à cette date (« hors ZUS » ou HZUS). Les deux équations de salaire s'écrivent comme suit :

$$
\begin{aligned}
& \operatorname{Ln} W^{\text {HZUS }}=\beta^{\text {HZUS }} X_{i}^{\text {HZUS }}+u_{i}^{\text {HZUS }} \\
& \operatorname{Ln} W^{Z U S}=\beta^{Z U S} X_{i}^{Z U S}+u_{i}^{Z U S}
\end{aligned}
$$

La décomposition d'Oaxaca (1973) ou celle proposée par Blinder la même année s'obtient à partir de la soustraction des salaires estimés séparément pour ces deux groupes d'individus, à savoir :

$$
\begin{aligned}
& \operatorname{Ln} \bar{W}^{\text {HZUS }}-\operatorname{Ln} \bar{W}^{Z U S} \\
& =\hat{\beta}^{\text {HZS }} \bar{X}^{\text {HZUS }}-\hat{\beta}^{\text {ZUS }} \bar{X}^{\text {ZUS }}-\hat{\beta}^{\text {HZUS }} \bar{X}^{\text {ZUS }}+\hat{\beta}^{\text {HzUS }} \bar{X}^{\text {ZUS }}
\end{aligned}
$$

ou encore

$$
\begin{aligned}
& \operatorname{Ln} \bar{W}^{\text {HZUS }}-\operatorname{Ln} \bar{W}^{Z U S} \\
& =\hat{\beta}^{\text {HZuS }}\left(\bar{X}^{\text {HZUS }}-\bar{X}^{\text {ZUS }}\right)+\left(\hat{\beta}^{\text {HzUS }}-\hat{\beta}^{\text {zUS }}\right) \bar{X}^{\text {ZUS }}
\end{aligned}
$$

Le premier terme représente la part du différentiel total de salaire moyen attribuable aux différences de caractéristiques moyennes entre les deux groupes "ZUS » et "Hors ZUS ", le second terme correspond à la différence de valorisation de ces caractéristiques (estimée aux caractéristiques moyennes de la sous-population en « ZUS ». Ce dernier terme s'annule quand les valorisations (les rendements des caractéristiques) sont identiques entre les deux sous-populations.

\section{Une norme de référence constituée par l'ensemble} de la population étudiée

Une des difficultés bien connue dans la mise en œuvre de la démarche de décomposition consiste dans le choix de la norme par laquelle valoriser l'écart des caractéristiques. Dans la formulation ci-dessus, ce sont les rendements des jeunes « hors ZUS » qui sont utilisés comme norme. Idéalement, il nous faudrait disposer d'une structure des salaires qui s'établirait en l'absence de toute discrimination afin d'évaluer les rendements tirés de chaque caractéristique. À défaut, nous suivons ici la proposition de Oaxaca et Ransom (1994) qui considère que la structure des salaires estimée pour l'ensemble de la population étudiée peut s'interpréter comme la norme non discriminante. II s'agit donc de prendre pour référence les coefficients de l'équation de salaire pour notre échantillon total regroupant les individus résidant dans une ZUS et ceux en dehors.

$$
\operatorname{Ln} W^{\text {HzusetzuS }}=\beta^{*} X_{i}^{\text {HzuSetzus }}+u_{i}^{\text {Hzusetzus }}
$$

$\hat{\beta}^{*}$ est alors l'ensemble des coefficients de l'équation de salaire estimée pour l'ensemble des jeunes quel que soit leur lieu de résidence et il constitue la norme non discriminante de valorisation des caractéristiques des individus. C'est en écart à ces valeurs qu'apparaîtront les valorisations différentielles des caractéristiques des individus.

En tenant compte de :

$\hat{\beta}^{*}\left(\bar{X}^{H Z U S}-\bar{X}^{Z U S}\right)-\bar{X}^{H Z U S} \hat{\beta}^{*}+\bar{X}^{Z U S} \hat{\beta}^{*}=0$

L'écart salarial moyen précédemment écrit devient :

$$
\begin{aligned}
& \operatorname{Ln} \bar{W}^{\text {HZUS }}-\operatorname{Ln} \bar{W}^{Z U S} \\
& =\hat{\beta}^{\text {HZUS }}\left(\bar{X}^{\text {HZUS }}-\bar{X}^{Z U S}\right)+\left(\hat{\beta}^{\text {HZUS }}-\hat{\beta}^{Z U S}\right) \bar{X}^{Z U S} \\
& +\hat{\beta}^{*}\left(\bar{X}^{\text {HZUS }}-\bar{X}^{Z U S}\right)-\hat{\beta}^{*} \bar{X}^{\text {HZUS }}+\hat{\beta}^{*} \bar{X}^{Z U S}
\end{aligned}
$$

On a :

$$
\begin{aligned}
& \operatorname{Ln} \bar{W}^{\text {HZUS }}-\operatorname{Ln} \bar{W}^{\text {ZUS }} \\
& =\hat{\beta}^{*}\left(\bar{X}^{\text {HzUs }}-\bar{X}^{\text {zus }}\right)+\left(\hat{\beta}^{\text {Hzus }}-\hat{\beta} \cdot\right) \bar{X}^{\text {Hzus }}+\left(\hat{\beta}^{*}-\hat{\beta}^{\text {zus }}\right) \bar{X}^{\text {zus }}
\end{aligned}
$$

Le premier terme de cette dernière formulation correspond aux différences de caractéristiques, évaluées " normalement ". Le second correspond à l'écart de valorisation des caractéristiques, pour le groupe d'individus résidant hors d'une ZUS. Le troisième terme correspond à ce même écart, mais pour le groupe des individus résidant en ZUS. Ce dernier est susceptible de traduire la discrimination salariale à l'encontre des habitants des ZUS, au motif de leur lieu de résidence.

\section{Introduire les différences d'allocation dans l'emploi}

La précédente décomposition peut être établie pour une catégorie d'emploi donnée ou pour l'ensemble des emplois mais en introduisant, parmi les variables explicatives des équations de salaire, les caractéristiques de ces emplois. Cependant, elle ne tient pas compte du fait que des barrières à l'entrée de certaines catégories d'emploi peuvent exister, notamment au détriment des jeunes résidant en ZUS comparativement aux autres jeunes urbains. 
Les salaires obtenus en moyenne par les jeunes résidant en ZUS, tout comme ceux hors d'une ZUS, dépendent des catégories des emplois qu'ils occupent ainsi que des probabilités d'accès à ces différentes catégories. On a :

$$
\begin{aligned}
& \bar{W}^{\text {HZUS }}=\sum_{j} P_{j}^{\text {HZUS }} \bar{W}_{j}^{\text {HZUS }} \text { avec } \sum_{j} P_{j}^{\text {HZUS }}=1 \\
& \bar{W}^{Z U S}=\sum_{j} P_{j}^{Z U S} \bar{W}_{j}^{Z U S} \text { avec } \sum_{j} P_{j}^{Z U S}=1
\end{aligned}
$$

$P_{j}^{Z U S}$ et $P_{j}^{H Z U S}$ représentent respectivement les pourcentages de jeunes de ZUS et hors de ZUS dans la catégorie d'emploi $j$. L'indice $j$ est ici relatif aux catégories retenues dans l'article à savoir les ouvriers non qualifiés, les ouvriers qualifiés, les employés non qualifiés, les employés qualifiés, les professions intermédiaires et les cadres (il pourrait s'agir aussi des diverses unités de production de l'économie, branches ou entreprises, etc.).

Le différentiel de salaire moyen dépend des allocations dans les catégories d'emploi, de sorte que l'on a :

$$
\bar{W}^{H Z U S}-\bar{W}^{Z U S}=\sum_{j} P_{j}^{H Z U S} \bar{W}_{j}^{H Z U S}-\sum_{j} P_{j}^{Z U S} \bar{W}_{j}^{Z U S}
$$

II se transforme ainsi :

$$
\begin{aligned}
& \bar{W}^{\text {HZUS }}-\bar{W}^{Z U S}=\sum_{j} P_{j}^{H Z U S} \bar{W}_{j}^{H Z U S}-\sum_{j} P_{j}^{Z U S} \bar{W}_{j}^{Z U S} \\
& +\sum_{j} P_{j}^{Z U S} \bar{W}_{j}^{\text {HZUS }}-\sum_{j} P_{j}^{Z U S} \bar{W}_{j}^{H Z U S} \\
& \bar{W}^{\text {HZUS }}-\bar{W}^{Z U S}=\sum_{j} P_{j}^{Z U S}\left(\bar{W}_{j}^{\text {HZUS }}-\bar{W}_{j}^{Z U S}\right) \\
& +\sum_{j} \bar{W}_{j}^{\text {HZUS }}\left(P_{j}^{\text {HZUS }}-P_{j}^{Z U S}\right)
\end{aligned}
$$

\section{La répartition des individus entre les différentes caté- gories d'emploi peut résulter de discriminations}

La catégorie d'emploi occupé différencie donc les populations sur le marché du travail et l'attribution des salaires. Les méthodes de décomposition standard jusqu'ici englobent ces inégalités d'accès à certaines catégories d'emploi dans la part de l'écart salarial attribuable aux différences de caractéristiques, empêchant d'isoler ce qui est dû à de la discrimination dans la structure d'emploi différente. Précisément, les calculs précédents ne prennent pas en compte le fait que la répartition des jeunes issus des "ZUS » dans les diverses catégories d'emploi peut, pour partie, résulter de traitements discriminatoires à leur encontre. II s'agit, à partir d'une régression logistique multinomiale (cf. tableau 6), d'estimer les probabilités d'accès aux catégories d'emplois pour les jeunes résidant hors d'une ZUS à partir d'un ensemble de caractéristiques individuelles, puis de simuler la distribution des emplois pour les jeunes résidant en ZUS comme s'ils possédaient la même structure d'accès aux emplois (si leurs caractéristiques recevaient la même valorisation dans l'accès aux différents emplois) que ceux habitant hors d'une ZUS.
La probabilité d'occuper un emploi de catégorie $j$ pour l'individu $i$ dépend de ses caractéristiques $Z_{i}$ et s'écrit :

$$
P_{i j}=\frac{e^{Z_{i} \theta_{j}}}{\sum_{k=1}^{J} e^{Z_{i} \theta_{k}}}
$$

où $\theta j$ est le vecteur des paramètres associés aux facteurs explicatifs de l'appartenance à la catégorie d'emploi $j$.

Soit $\hat{P}_{j}^{Z U S}$, la proportion hypothétique de jeunes résidant en ZUS qui seraient dans la catégorie d'emploi $j$ s'ils faisaient face aux mêmes mécanismes d'allocation dans les emplois que ceux habitant hors d'une ZUS. Elle s'obtient de la manière suivante :

$\hat{P}_{j}^{Z U S}=\frac{1}{N_{Z U S}} \sum_{i \in Z \cup S}\left(\frac{e^{Z_{j}^{\text {ZUS }} \hat{\theta}_{j}^{\text {HZUS }}}}{\sum_{j=1}^{J} e^{Z_{j}^{\text {ZUS }} \hat{\theta}_{j}^{\text {HZUS }}}}\right)$ avec $\sum_{j} \hat{P}_{j}^{Z U S} 1$

où $N_{\text {zuS }}$ est le nombre d'individus dans les ZUS.

En introduisant ces probabilités hypothétiques dans la décomposition précédente, on a :

$$
\begin{aligned}
& \bar{W}^{\text {HZUS }}-\bar{W}^{Z U S}=\sum_{j} P_{j}^{Z U S}\left(\bar{W}_{j}^{\text {HZUS }}-\bar{W}_{j}^{Z U S}\right) \\
& +\sum_{j} \bar{W}_{j}^{\text {HZUS }}\left(P_{j}^{\text {HZUS }}-\hat{P}_{j}^{Z U S}+\hat{P}_{j}^{Z U S}-P_{j}^{Z U S}\right) \\
& \bar{W}^{\text {HZUS }}-\bar{W}^{Z U S}=\sum_{j} P_{j}^{Z U S}\left(\bar{W}_{j}^{\text {HZUS }}-\bar{W}_{j}^{Z U S}\right) \\
& +\sum_{j} \bar{W}_{j}^{\text {HZUS }}\left(P_{j}^{\text {HZUS }}-\hat{P}_{j}^{Z U S}\right)+\sum_{j} \bar{W}_{j}^{\text {HZUS }}\left(\hat{P}_{j}^{Z U S}-P_{j}^{Z U S}\right)
\end{aligned}
$$

La décomposition de Brown, Moon et Zoloth (1980) identifie les composantes de l'écart salarial au sein de chaque catégorie d'emploi et les composantes de l'écart entre les catégories d'emploi

Au sein de chaque catégorie d'emploi $j$, les écarts de salaire se décomposent selon la méthode de Oaxaca et Ransom. On obtient alors la modalité précise de la décomposition de Brown, Moon et Zoloth, sur laquelle nous nous appuyons dans l'article.

$$
\begin{aligned}
& \bar{W}_{\text {HZUS }}-\bar{W}_{Z U S}=\sum_{j} P_{j}^{Z U S} \hat{\beta}_{j}^{*}\left(\bar{X}_{j}^{\text {HZUS }}-\bar{X}_{j}^{Z U S}\right) \\
& +\sum_{j} P_{j}^{Z U S} \bar{X}_{j}^{\text {HZUS }}\left(\hat{\beta}_{j}^{H Z U S}-\hat{\beta}_{j}^{*}\right)+ \\
& \sum_{j} P_{j}^{Z U S} \bar{X}_{j}^{Z U S}\left(\hat{\beta}_{j}^{*}-\hat{\beta}_{j}^{Z U S}\right)+\sum_{j} \bar{W}_{j}^{H Z U S}\left(P_{j}^{H Z U S}-\hat{P}_{j}^{Z U S}\right) \\
& +\sum_{j} \bar{W}_{j}^{H Z U S}\left(\hat{P}_{j}^{Z U S}-P_{j}^{Z U S}\right)
\end{aligned}
$$

où $\hat{\beta}_{j}^{\text {HZUS }}$ et $\hat{\beta}_{j}^{Z U S}$ sont les coefficients estimés des équations de salaires pour les jeunes résidant en ZUS et ceux résidant hors d'une ZUS, tandis que $\hat{\beta}_{j}^{*}$ est le vecteur des coefficients de l'équation de salaire « non discriminante ", pour les $j$ catégories d'emploi $(j=1, \ldots J$, ici $J=6$ ). 
ANNEXE 2

Tableau A

Équation de salaire pour la population des jeunes résidents d'une ZUS et hors d'une ZUS en 1998 Variable dépendante : le logarithme du salaire mensuel net, prime incluse, en euros, à l'embauche du premier emploi

\begin{tabular}{|c|c|c|c|c|}
\hline \multirow[b]{2}{*}{$\begin{array}{l}\text { Constante } \\
\text { Homme }\end{array}$} & \multicolumn{2}{|c|}{ Jeunes non issus de ZUS } & \multicolumn{2}{|c|}{ Jeunes issus de ZUS } \\
\hline & $\begin{array}{l}6,317^{\star \star \star} \\
0,069^{\star \star \star}\end{array}$ & $\begin{array}{l}(0,010) \\
(0,005)\end{array}$ & $\begin{array}{l}6,343^{\star \star \star} \\
0,050^{\star \star \star}\end{array}$ & $\begin{array}{l}(0,024) \\
(0,014)\end{array}$ \\
\hline $\begin{array}{l}\text { Temps de travail inférieur au temps plein } \\
\text { Travail à temps plein }\end{array}$ & $\begin{array}{l}\text { Réf. } \\
0,383^{\star \star \star}\end{array}$ & $(0,005)$ & $\begin{array}{l}\text { Réf. } \\
0,412^{\star \star *}\end{array}$ & $(0,014)$ \\
\hline $\begin{array}{l}\text { Diplôme } \\
\text { Sans diplôme } \\
\text { CAP-BEP } \\
\text { Baccalauréat } \\
\text { Baccalauréat }+2 \text { (BTS/DUT) } \\
\text { Baccalauréat }+3 \\
\text { Baccalauréat }+4 \text { et plus }\end{array}$ & $\begin{array}{c}\text { Réf. } \\
0,032^{\star \star \star} \\
0,041^{\star \star \star} \\
0,168^{\star \star \star} \\
0,096^{\star \star \star} \\
0,207^{\star \star \star}\end{array}$ & $\begin{array}{l}(0,008) \\
(0,008) \\
(0,009) \\
(0,011) \\
(0,010)\end{array}$ & $\begin{array}{l}\text { Réf. } \\
0,018 \\
0,040^{\star \star} \\
0,156^{\star \star \star} \\
0,053^{\star} \\
0,174^{\star \star \star}\end{array}$ & $\begin{array}{l}(0,017) \\
(0,019) \\
(0,023) \\
(0,032) \\
(0,027)\end{array}$ \\
\hline Spécialité industrielle & $0,018^{\star \star \star}$ & $(0,006)$ & $0,046^{\star \star \star}$ & $(0,015)$ \\
\hline $\begin{array}{l}\text { Catégorie d'emploi } \\
\text { Ouvrier non qualifié } \\
\text { Ouvrier qualifié } \\
\text { Employé non qualifié } \\
\text { Employé qualifié } \\
\text { Profession intermédiaire } \\
\text { Cadre }\end{array}$ & $\begin{array}{c}\text { Réf. } \\
0,012 \\
-0,076^{\star \star \star} \\
0,008 \\
0,104^{\star \star \star} \\
0,397^{\star \star \star}\end{array}$ & $\begin{array}{r}(0,009) \\
(0,008) \\
(0,009) \\
(0,008) \\
0,010)\end{array}$ & $\begin{array}{c}\text { Réf. } \\
-0,006 \\
-0,060^{\star \star \star} \\
-0,024 \\
0,079^{\star \star *} \\
0,382^{\star \star *}\end{array}$ & $\begin{array}{l}(0,020) \\
(0,019) \\
(0,022) \\
(0,021) \\
(0,032)\end{array}$ \\
\hline Avoir effectué au moins un stage durant ses études & $0,020^{\star \star \star}$ & $(0,004)$ & 0,006 & $(0,012)$ \\
\hline $\begin{array}{l}\text { Lieu de naissance des parents } \\
\text { Les deux parents nés en France ou de nationalité } \\
\text { française } \\
\text { Un des } 2 \text { parents ou les } 2 \text { nés hors de France }\end{array}$ & $\begin{array}{c}\text { Réf. } \\
0,005\end{array}$ & $(0,005)$ & $\begin{array}{r}\text { Réf. } \\
-0,015\end{array}$ & $(0,012)$ \\
\hline $\begin{array}{l}\text { Ratio (Cadres + Professions intermédiaires) / } \\
\text { (Employés + Ouvriers) }\end{array}$ & $0,00015^{\star \star \star}$ & $(0,00003)$ & 0,0003 & $(0,0002)$ \\
\hline $\begin{array}{l}\text { Résider hors île-de-France à la fin des études } \\
\text { Résider en île-de-France à la fin des études }\end{array}$ & $\begin{array}{l}\text { Réf. } \\
0,049^{\star \star *}\end{array}$ & $(0,005)$ & $\begin{array}{l}\text { Réf. } \\
0,067^{\star \star \star}\end{array}$ & $(0,015)$ \\
\hline $\begin{array}{l}R^{2} \text { ajusté (en \%) } \\
\text { Effectifs }\end{array}$ & \multicolumn{2}{|c|}{$\begin{array}{c}50,5 \\
20492\end{array}$} & \multicolumn{2}{|c|}{$\begin{array}{c}44,9 \\
2525\end{array}$} \\
\hline
\end{tabular}

Lecture : les seuils de significativité sont respectivement égaux à $1 \%\left(^{* * *}\right), 5 \%\left(^{* *}\right)$ et $10 \%\left(^{*}\right)$. Les écarts-types figurent entre parenthèses.

Champ : jeunes sortis du système éducatif en 1998 résidant en ZUS ou dans une agglomération contenant une ZUS, ayant eu au moins un emploi au cours de leurs trois premières années de vie active.

Source : enquête Génération 98, première interrogation, Céreq. 
Tableau B

L'accès aux différentes catégories d'emploi (ensemble des jeunes)

Régression logistique multinomiale - Référence : ouvriers non qualifiés

\begin{tabular}{|c|c|c|c|c|c|c|}
\hline & $\begin{array}{c}\text { Ouvriers } \\
\text { non qualifiés }\end{array}$ & $\begin{array}{l}\text { Ouvriers } \\
\text { qualifiés }\end{array}$ & $\begin{array}{c}\text { Employés } \\
\text { non qualifiés }\end{array}$ & $\begin{array}{l}\text { Employés } \\
\text { qualifiés }\end{array}$ & $\begin{array}{l}\text { Profession } \\
\text { Intermé- } \\
\text { diaire }\end{array}$ & Cadres \\
\hline Constante & - & $\begin{array}{c}-1,432^{\star \star \star} \\
(0,096)\end{array}$ & $\begin{array}{l}0,811^{\star \star \star} \\
(0,076)\end{array}$ & $\begin{array}{c}-0,668^{\star \star \star} \\
(0,101)\end{array}$ & $\begin{array}{c}-0,986^{\star \star \star} \\
(0,098)\end{array}$ & $\begin{array}{c}-4,655^{\star \star \star} \\
(0,330)\end{array}$ \\
\hline $\begin{array}{l}\text { Femme } \\
\text { Homme }\end{array}$ & - & $\begin{array}{c}\text { Réf. } \\
0,497^{\star \star \star} \\
(0,082)\end{array}$ & $\begin{array}{c}\text { Réf. } \\
-1,582^{\star \star \star} \\
(0,060)\end{array}$ & $\begin{array}{c}\text { Réf. } \\
-1,388^{\star \star \star} \\
(0,065)\end{array}$ & $\begin{array}{c}\text { Réf. } \\
-0,905^{\star \star \star} \\
(0,060)\end{array}$ & $\begin{array}{c}\text { Réf. } \\
-0,327^{\star \star \star} \\
(0,072)\end{array}$ \\
\hline $\begin{array}{l}\text { Diplôme } \\
\text { Sans diplôme } \\
\text { CAP-BEP } \\
\text { Baccalauréat } \\
\text { Baccalauréat + } 2 \text { (BTS/DUT) } \\
\text { Baccalauréat + } 3 \\
\text { Baccalauréat }+4 \text { et plus }\end{array}$ & $\begin{array}{l}- \\
- \\
- \\
- \\
-\end{array}$ & $\begin{array}{l}\text { Réf. } \\
0,550^{\star \star \star} \\
(0,068) \\
0,295^{\star \star \star} \\
(0,080) \\
0,163 \\
(0,108) \\
0,465^{\star \star} \\
(0,228) \\
0,280 \\
(0,189)\end{array}$ & $\begin{array}{l}\text { Réf. } \\
0,236^{\star \star \star} \\
(0,070) \\
0,288^{\star \star \star} \\
(0,074) \\
0,255^{\star \star \star} \\
(0,095) \\
0,891^{\star \star \star} \\
(0,162) \\
0,726^{\star \star \star} \\
(0,141)\end{array}$ & $\begin{array}{l}\text { Réf. } \\
0,884^{\star \star \star} \\
(0,100) \\
1,509^{\star \star \star} \\
(0,098) \\
2,302^{\star \star \star} \\
(0,109) \\
2,375^{\star \star \star} \\
(0,172) \\
2,970^{\star \star \star} \\
(0,145)\end{array}$ & $\begin{array}{l}\text { Réf. } \\
0,302^{\star \star \star} \\
(0,105) \\
1,717^{\star \star \star} \\
(0,094) \\
3,513^{\star \star \star} \\
(0,100) \\
3,679^{\star \star \star} \\
(0,161) \\
4,038^{\star \star \star} \\
(0,138)\end{array}$ & $\begin{array}{c}\text { Réf. } \\
0,242 \\
(0,431) \\
1,905^{\star \star \star} \\
(0,351) \\
3,786^{\star \star \star} \\
(0,334) \\
6,132^{\star \star \star} \\
(0,352) \\
8,053^{\star \star \star} \\
(0,338)\end{array}$ \\
\hline $\begin{array}{l}\text { Autres spécialités } \\
\text { Spécialité industrielle }\end{array}$ & - & $\begin{array}{l}\text { Réf. } \\
0,430^{\star \star \star} \\
(0,064)\end{array}$ & $\begin{array}{c}\text { Réf. } \\
-1,138^{\star * *} \\
(0,064)\end{array}$ & $\begin{array}{c}\text { Réf. } \\
-1,457^{\star \star *} \\
(0,077)\end{array}$ & $\begin{array}{c}\text { Réf. } \\
-0,937^{\star \star \star} \\
(0,062)\end{array}$ & $\begin{array}{c}\text { Réf. } \\
-0,341^{\text {*** }} \\
(0,083)\end{array}$ \\
\hline $\begin{array}{l}\text { Profession du père } \\
\text { Père ouvrier, employé, autres } \\
\text { Père artisan, commerçant } \\
\text { Père profession intermédiaire } \\
\text { Père cadre }\end{array}$ & $\begin{array}{l}- \\
-\end{array}$ & $\begin{array}{l}\text { Réf. } \\
0,264^{\star \star \star} \\
(0,096) \\
0,011 \\
(0,062) \\
0,161 \\
(0,102)\end{array}$ & $\begin{array}{l}\text { Réf. } \\
0,430^{\star \star \star} \\
(0,093) \\
0,250^{\star \star \star} \\
(0,060) \\
0,527^{\star \star \star} \\
(0,092)\end{array}$ & $\begin{array}{l}\text { Réf. } \\
0,399^{\star \star \star} \\
(0,102) \\
0,311^{\star \star \star} \\
(0,066) \\
0,641^{\star \star \star} \\
(0,094)\end{array}$ & $\begin{array}{l}\text { Réf. } \\
0,551^{\star \star \star} \\
(0,092) \\
0,234^{\star \star \star} \\
(0,061) \\
0,747^{\star \star \star} \\
(0,086)\end{array}$ & $\begin{array}{l}\text { Réf. } \\
0,575^{\text {} * \star} \\
(0,117) \\
0,138 \\
(0,085) \\
1,131^{\star * \star} \\
(0,101)\end{array}$ \\
\hline $\begin{array}{l}\text { Lieu de naissance des parents } \\
\text { Les deux parents nés en France ou de } \\
\text { nationalité française } \\
\text { Un des } 2 \text { parents ou les } 2 \text { né(s) hors } \\
\text { de France }\end{array}$ & - & $\begin{array}{c}\text { Réf. } \\
-0,141^{\star *} \\
(0,063)\end{array}$ & $\begin{array}{c}\text { Réf. } \\
0,002 \\
(0,060)\end{array}$ & $\begin{array}{c}\text { Réf. } \\
-0,198^{\text {*** }} \\
(0,068)\end{array}$ & $\begin{array}{c}\text { Réf. } \\
-0,166^{\star \star \star} \\
(0,063)\end{array}$ & $\begin{array}{c}\text { Réf. } \\
-0,220^{\star *} \\
(0,086)\end{array}$ \\
\hline $\begin{array}{l}\text { Résider hors île-de-France à la fin des } \\
\text { études } \\
\text { Résider en Île-de-France à la fin des } \\
\text { études }\end{array}$ & - & $\begin{array}{l}\text { Réf. } \\
0,207^{\star \star \star} \\
(0,070)\end{array}$ & $\begin{array}{l}\text { Réf. } \\
0,461^{\star \star \star} \\
(0,066)\end{array}$ & $\begin{array}{l}\text { Réf. } \\
0,698^{\star \star \star} \\
(0,070)\end{array}$ & $\begin{array}{l}\text { Réf. } \\
0,675^{\star \star \star} \\
(0,066)\end{array}$ & $\begin{array}{l}\text { Réf. } \\
0,897^{\star \star \star} \\
(0,079)\end{array}$ \\
\hline $\begin{array}{l}\text { Ne pas habiter en ZUS à la fin des } \\
\text { études } \\
\text { Habiter en ZUS à la sortie des } \\
\text { études }\end{array}$ & - & $\begin{array}{c}\text { Réf. } \\
-0,107 \\
(0,077)\end{array}$ & $\begin{array}{c}\text { Réf. } \\
-0,263 \\
(0,074)\end{array}$ & $\begin{array}{l}\text { Réf. } \\
-0,234^{\star \star \star} \\
(0,085)\end{array}$ & $\begin{array}{l}\text { Réf. } \\
-0,171^{\text {** }} \\
(0,079)\end{array}$ & $\begin{array}{l}\text { Réf. } \\
-0,454^{\star \star \star} \\
(0,119)\end{array}$ \\
\hline $\begin{array}{l}\text { Log-Vraisemblance } \\
\text { Nombre d'observations } \\
\text { Statistique } L R \\
P \text {-Value } \\
\text { Pseudo } R^{2}\end{array}$ & \multicolumn{6}{|c|}{$\begin{array}{c}-28859 \\
23017 \\
22591 \\
0,000\end{array}$} \\
\hline
\end{tabular}

Lecture : régression logistique multinomiale. Les seuils de significativité sont respectivement égaux à $1 \%\left(^{* * *}\right)$, $5 \%\left(^{* *}\right)$ et $10 \%\left({ }^{*}\right)$. Les écarts-types figurent entre parenthèses. La modalité d'accès à l'emploi de référence est celle qui consiste à devenir ouvrier non qualifié. Les caractéristiques individuelles de référence sont les suivantes : femme sans diplôme, ayant suivi une formation générale ou tertiaire, de père ouvrier ou employé, ayant les deux parents nés en France ou de nationalité française, résidant hors d'île-de-France à la fin des études.

Relativement aux caractéristiques de ceux qui deviennent ouvriers non qualifiés, les jeunes résidant en ZUS qui deviennent employés qualifiés sont moins souvent des hommes, le coefficient associé à cette variable étant statistiquement significativement positif, au seuil de $1 \%$.

Champ : jeunes sortis du système éducatif en 1998 résidant en ZUS ou dans une unité urbaine contenant une ZUS, ayant eu au moins un emploi au cours de leurs trois premières années de vie active.

Source : enquête Génération 98, première interrogation, Céreq. 
Tableau C

Équation de salaire pour l'ensemble des jeunes à l'embauche du premier emploi

\begin{tabular}{|c|c|c|}
\hline \multirow[b]{2}{*}{$\begin{array}{l}\text { Constante } \\
\text { Homme }\end{array}$} & \multicolumn{2}{|c|}{$\begin{array}{l}\text { Ensemble des jeunes résidant en ZUS } \\
\text { ou dans une unité urbaine contenant une ZUS } \\
\text { à leur sortie du système éducatif }\end{array}$} \\
\hline & $\begin{array}{l}6,278^{\star \star \star} \\
0,095^{\star \star \star}\end{array}$ & $\begin{array}{l}(0,008) \\
(0,005)\end{array}$ \\
\hline $\begin{array}{l}\text { Temps de travail inférieur au temps plein } \\
\text { Travail à temps plein }\end{array}$ & $\begin{array}{l}\text { Réf. } \\
0,412^{\star \star \star}\end{array}$ & $(0,005)$ \\
\hline $\begin{array}{l}\text { Diplôme } \\
\text { Sans diplôme } \\
\text { CAP-BEP } \\
\text { Baccalauréat } \\
\text { Baccalauréat }+2 \text { (BTS/DUT) } \\
\text { Baccalauréat }+3 \\
\text { Baccalauréat }+4 \text { et plus }\end{array}$ & $\begin{array}{c}\text { Réf. } \\
0,030^{\star \star \star} \\
0,068^{\star \star \star} \\
0,245^{\star \star \star} \\
0,233^{\star \star \star} \\
0,483^{\star \star \star}\end{array}$ & $\begin{array}{l}(0,008) \\
(0,008) \\
(0,008) \\
(0,010) \\
(0,008)\end{array}$ \\
\hline Spécialité industrielle & $0,027^{\star \star \star}$ & $(0,005)$ \\
\hline Avoir effectué au moins un stage durant ses études & $0,023^{\star \star \star}$ & $(0,004)$ \\
\hline $\begin{array}{l}\text { Lieu de naissance des parents } \\
\text { Les deux parents nés en France ou de nationalité française } \\
\text { Un des } 2 \text { parents ou les } 2 \text { nés hors de France }\end{array}$ & $\begin{array}{r}\text { Réf. } \\
-0,005\end{array}$ & $(0,005)$ \\
\hline $\begin{array}{l}\text { Résider hors île-de-France à la fin des études } \\
\text { Résider en île-de-France à la fin des études }\end{array}$ & $\begin{array}{c}\text { Réf. } \\
0,069\end{array}$ & $(0,005)$ \\
\hline $\begin{array}{l}\text { Résider hors ZUS à la fin des études } \\
\text { Résider en ZUS }\end{array}$ & $\begin{array}{l}\text { Réf. } \\
-0,014^{\star \star}\end{array}$ & $(0,007)$ \\
\hline $\begin{array}{l}R^{2} \text { ajusté (en \%) } \\
\text { Effectifs }\end{array}$ & \multicolumn{2}{|c|}{$\begin{array}{c}43,5 \\
23017\end{array}$} \\
\hline
\end{tabular}

Lecture : les seuils de significativité sont respectivement égaux à $1 \%\left(^{* * *}\right), 5 \%\left(^{* *}\right)$ et $10 \%\left(^{*}\right)$. Les écarts-types figurent entre parenthèses.

Champ : jeunes sortis du système éducatif en 1998 résidant en ZUS ou dans une unité urbaine contenant une ZUS, ayant eu au moins un emploi au cours de leurs trois premières années de vie active.

Source : enquête Génération 98, première interrogation, Céreq. 